\title{
Maximum Score Estimation of Preference Parameters for a Binary Choice Model under Uncertainty *
}

\author{
Le-Yu Chen \\ Institute of Economics, Academia Sinica \\ Sokbae Lee \\ Department of Economics, Seoul National University \\ Myung Jae Sung ${ }^{\dagger}$ \\ School of Economics, Hongik University
}

1 December 2013

\begin{abstract}
This paper develops maximum score estimation of preference parameters in the binary choice model under uncertainty in which the decision rule is affected by conditional expectations. The preference parameters are estimated in two stages: we estimate conditional expectations nonparametrically in the first stage and then the preference parameters in the second stage based on Manski $(1975,1985)$ 's maximum score estimator using the choice data and first stage estimates. The paper establishes consistency and derives rate of convergence of the two-stage maximum score estimator. Moreover, the paper also provides sufficient conditions under which the two-stage estimator is asymptotically equivalent in distribution to the corresponding single-stage estimator
\end{abstract}

*This work was in part supported by National Science Council of Taiwan (102-2410-H-001-012), by European Research Council (ERC-2009-StG-240910- ROMETA) and by the National Research Foundation of Korea Grant funded by the Korean Government (NRF-2012-S1A3-A2033467). We thank Hidehiko Ichimura, Liangjun Su, participants at 2013 Asian Meeting of the Econometric Society and seminar participants at Singapore Management University, a co-editor and three anonymous referees for helpful comments on this work.

$\dagger$ Corresponding author. Address: School of Economics, Hongik University, 94 Wausan-ro, MapoGu, Seoul, South Korea 121-791. E-mail: mjaesung@hongik.ac.kr. 
that assumes the first stage input is known. These results are of independent interest for maximum score estimation with nonparametrically generated regressors. The paper also presents some Monte Carlo simulation results for finite-sample behavior of the two-stage estimator.

Keywords: discrete choice, maximum score estimation, generated regressor, preference parameters, M-estimation, cube root asymptotics

JEL Codes: C12, C13, C14.

\section{Introduction}

This paper develops a semiparametric two-stage estimator of preference parameters in the binary choice model where the agent's decision rule is affected by conditional expectations of outcomes which are uncertain at the choice-making stage and the preference shocks are nonparametrically distributed with unknown form of heteroskedasticity. The pioneering papers of Manski $(1991,1993)$ established nonparametric identification of agents' expectations in the discrete choice model under uncertainty when the expectations are fulfilled and conditioned only on observable variables. Utilizing this result, Ahn and Manski (1993) proposed a two-stage estimator for a binary choice model under uncertainty where agent's utility was linear in parameter and the unobserved preference shock had a known distribution. Specifically, they estimated the agent's expectations nonparametrically in the first stage and then the preference parameters in the second stage by maximum likelihood estimation using the choice data and the expectation estimates. Ahn $(1995,1997)$ extended the two-step approach further. On one hand, Ahn (1995) considered nonparametric estimation of conditional choice probabilities in the second stage. On the other hand, Ahn (1997) retained the linear index structure of the Ahn-Manski model but estimated the preference parameters in the second stage using average derivative method hence allowing for unknown distribution of the unobservable. In principle, alternative approaches accounting for nonparametric unobserved preference shock can also be applied in the second step estimation of this framework. Well known methods include Cosslett (1983), Powell et al. (1989), Ichimura (1993), Klein and Spady (1993), and Coppejans (2001), among many others. 
The aforementioned papers allow for nonparametric setting of the distribution of the preference shock. But the unobserved shock is assumed either to be independent of or to have specific dependence structure with the covariates. By contrast, Manski $(1975,1985)$ considered a binary choice model under the conditional median restriction and thus allowed for general form of heteroskedasticity for the unobserved shock. It is particularly important, as shown in Brown and Walker (1989), to account for heteroskedasticity in random utility models. Therefore, this paper develops the semiparametric two-stage estimation method for the Ahn-Manski model where the second stage is based on Manski $(1975,1985)$ 's maximum score estimator and thus can accommodate nonparametric preference shock with unknown form of heteroskedasticity.

From a methodological perspective, this paper also contributes to the literature on two-stage M-estimation method with non-smooth criterion functions. When the true parameter value can be formulated as the unique root of certain population moment equations, the problem of M-estimation can be reduced to that of Z-estimation. Chen et al. (2003) considered semiparametric non-smooth Z-estimation problem with estimated nuisance parameter, while allowing for over-identifying restrictions. Chen and Pouzo (2009,2012) developed general estimation methods for semiparametric and nonparametric conditional moment models with possibly non-smooth generalized residuals. For the general M-estimation problem, Ichimura and Lee (2010) assumed some degree of second-order expansion of the underlying objective function and established conditions under which one can obtain a $\sqrt{N}$-consistent estimator of the finite dimensional parameter where $N$ is the sample size when the nuisance parameter at the first stage is estimated at a slower rate. For more recent papers on two-step semiparametric estimation, see Ackerberg et al. (2012), Chen et al. (2013), Escanciano et al. (2012, 2013), Hahn and Ridder (2013), and Mammen et al. (2013), among others. None of the aforementioned papers include the maximum score estimation in the second stage estimation.

For this paper, the second stage maximum score estimation problem cannot be reformulated as a Z-estimation problem. Furthermore, even in the absence of nui- 
sance parameter, Kim and Pollard (1990) demonstrated that the maximum score estimator can only have the cube root rate of convergence and its asymptotic distribution is non-standard. The most closely related paper is Lee and Pun (2006) who showed that $m$ out of $n$ bootstrapping can be used to consistently estimate sampling distributions of nonstandard M-estimators with nuisance parameters. Their general framework includes the maximum score estimator as a special case, but allowing for only parametric nuisance parameters. Therefore, established results in the two-stage estimation literature are not immediately applicable and the asymptotic theory developed in this paper may also be of independent interest for non-smooth M-estimation with nonparametrically generated covariates.

The rest of the paper is organized as follows. Section 2 sets up the binary choice model under uncertainty and presents the two-stage maximum score estimation procedure of the preference parameters. Section 3 states regularity assumptions and derives consistency and rate of convergence of the estimator. In addition, Section 3 gives conditions under which the two-stage maximum score estimator is asymptotically equivalent to the infeasible single-stage maximum score estimator with a known first stage input. Section 4 presents Monte Carlo studies assessing finite sample performance of the estimator. Section 5 gives further applications of maximum score estimation with nonparametrically generated regressors. Section 6 concludes this paper. Proofs of technical results along with some preliminary lemmas are given in the Appendices.

\section{Maximum Score Estimation of a Binary Choice Model under Uncertainty}

Suppose an agent must choose between two actions denoted by 0 and 1 . The utility from choosing action $j \in\{0,1\}$ is

$$
U_{j}=v_{j}^{\prime} \beta_{1}+y^{\prime} \beta_{2}+\varepsilon_{j}
$$


Realization of the random vector $\left(v_{j}, \varepsilon_{j}\right) \in R^{k} \times R$ is known to the agent before the action is chosen and the random vector $y \in R^{p}$ is realized only after the action is chosen. Random vectors $\left(v_{1}, \varepsilon_{1}\right)$ and $\left(v_{0}, \varepsilon_{0}\right)$ are not necessarily identical. Distribution of $y$ depends on the chosen action and realization of a random vector $x \in R^{q}$. Let $E^{s}(\cdot \mid \cdot)$ denote the agent's subjective conditional expectation. Given the realization of $\left(v_{j}, \varepsilon_{j}\right)$, the agent chooses the action $d$ that maximizes the expected utility:

$$
v_{j}^{\prime} \beta_{1}+E^{s}(y \mid x, d=j)^{\prime} \beta_{2}+\varepsilon_{j}, j \in\{0,1\} .
$$

Thus the decision rule has the form

$$
d=1\left\{z^{\prime} \beta_{1}+\left[E^{s}(y \mid x, d=1)-E^{s}(y \mid x, d=0)\right]^{\prime} \beta_{2}>\varepsilon\right\},
$$

where $z \equiv v_{1}-v_{0}, \varepsilon \equiv \varepsilon_{0}-\varepsilon_{1}$, and $1\{\cdot\}$ is an indicator function whose value is one if the argument is true and zero otherwise.

As in Ahn and Manski (1993), suppose that expectations are fulfilled:

$$
E^{s}(y \mid x, d=j)=E(y \mid x, d=j) .
$$

We assume that the researcher does not observe realization of $\varepsilon$ and $E(y \mid x, d=j)$, but that of $(z, x, d, y)$.

Let $G(x) \equiv E(y \mid x, d=1)-E(y \mid x, d=0)$ and let $w \equiv(z, G(x)) \in \mathcal{W} \subset R^{k+p}$, where $\mathcal{W}$ denotes the support of the distribution of $w$. Then, equation (2.1) can be written as

$$
d=1\left\{w^{\prime} \beta>\varepsilon\right\},
$$

where $\beta \equiv\left(\beta_{1}, \beta_{2}\right)$ is a vector of unknown preference parameters. The set of assumptions leading to the binary choice model in (2.2) is equivalent to that of Ahn and Manski (1993, equations (1)-(3)). Note that $x$ affects the agent's decision only through $G(x)$, and therefore, $x$ and $z$ can have common elements, as long as the support of the distribution of $w$ is not contained in any proper linear subspace of $R^{k+p}$. 
In this paper, we consider an important deviation from Ahn and Manski (1993)'s setup where the unobserved preference shock $\varepsilon$ is independent of $(z, x)$ with a known distribution function. Instead, we consider inference under a flexible specification of the unobserved model component. Following Manski (1985), we impose the restriction:

$$
\operatorname{Med}(\varepsilon \mid z, x)=0 .
$$

The conditional median independence assumption in (2.3) allows for heteroskedasticity of unknown form, and hence, is substantially weaker than the assumption imposed in Ahn and Manski (1993). Given (2.3), the model (2.1) then satisfies

$$
\operatorname{Med}(d \mid z, x)=1\left\{w^{\prime} \beta>0\right\} .
$$

We may consider sufficient conditions for (2.3) in terms of the original structural errors $\varepsilon_{0}$ and $\varepsilon_{1}$. Recall that $\varepsilon \equiv \varepsilon_{0}-\varepsilon_{1}$. Suppose that (i) the distribution of $\left(\varepsilon_{0}, \varepsilon_{1}\right)$ is the same as that of $\left(\varepsilon_{1}, \varepsilon_{0}\right)$ conditional on $x$ and $z$, and (ii) the support of this common conditional distribution is $R^{2}$. This type of condition is called conditional exchangeability assumption. Then this implies that $\varepsilon$ is symmetrically distributed around zero, thereby implying equation (2.3). For further discussions regarding conditional exchangeability assumption, see Fox (2007) in the context of multinomial discrete-choice models and Arellano and Honoré (2001) for applications in panel data models, among others. Also, note that the conditional exchangeability assumption is a sufficient (but not necessary) condition for equation (2.3).

Let $\Theta$ denote the space of preference parameters, and let $\Lambda_{j}, j \in\{1, \ldots, p\}$, denote the function space of difference of conditional expectations $E\left(y_{j} \mid x, d=1\right)-$ $E\left(y_{j} \mid x, d=0\right)$. Moreover, let $b \equiv\left(b_{1}, b_{2}\right)$ and $\gamma_{j}(x), j \in\{1, \ldots, p\}$, denote generic elements of $\Theta$ and $\Lambda_{j}$, respectively. Let $\gamma(x) \equiv\left(\gamma_{1}(x), \ldots, \gamma_{p}(x)\right)$ and $\Lambda \equiv \prod_{j=1}^{p} \Lambda_{j}$ be the space of $\gamma$. We refer to $\beta \equiv\left(\beta_{1}, \beta_{2}\right)$ and $G(x)$ as the true finite-dimensional and infinite-dimensional parameters.

Suppose that data consist of random samples $\left(z_{i}, x_{i}, d_{i}, y_{i}\right), i=1, \cdots, N$. We estimate in the first stage the conditional expectations which are not observed. Let 
$\widehat{G}\left(x_{i}\right)$ denote an estimate of the difference in conditional expectations. Using the

estimate $\widehat{G}$, we estimate the preference parameters $\beta$ in the second stage by the method of maximum score estimation of Manski $(1975,1985)$. For any $b$ and $\gamma$, define the sample score function

$$
S_{N}(b, \gamma) \equiv \frac{1}{N} \sum_{i=1}^{N} \tau_{i}\left(2 d_{i}-1\right) 1\left\{z_{i}^{\prime} b_{1}+\gamma\left(x_{i}\right)^{\prime} b_{2}>0\right\},
$$

where $\tau_{i} \equiv \tau\left(x_{i}\right)$ is a predetermined weight function to avoid unduly influences from estimated $G\left(x_{i}\right)$ at data points carrying low density. The two-stage estimator of $\beta$ is now defined as

$$
\widehat{\beta}=\arg \max _{b \in \Theta} \mathrm{S}_{N}(b, \widehat{G}) .
$$

We end this section by commenting on inherent features of the maximum score estimation approach. The zero conditional median assumption does not require the existence of any error moments and allows heteroskedastic errors of an unknown form. However, the maximum score approach has its drawbacks, mainly due to its weak assumption. First, in terms of prediction power, it can identify unknown parameters up to scale and also only identify whether the conditional probability of $d=1$ is above or below one half; hence, the partial effects of covariates are not identified. Second, lack of smoothness in the objective function makes computation of the estimator difficult and lets the estimator converge in probability to the true parameter at a rate of $N^{-1 / 3}$.

\section{Consistency, Rate of Convergence and Asymp- totic Distribution of $\widehat{\beta}$}

Let $F(t ; b)$ and $f(t ; b)$, respectively, denote the distribution and density of $w^{\prime} b$. To simplify the analysis, we consider fixed trimming such that $\tau(x)=1(x \in \mathcal{X})$, where $\mathcal{X} \subset \mathcal{R}^{q}$ is a predetermined, compact, and convex subset of the support of $x$. For any real vector $b$, let $\|b\|_{E}$ denote the Euclidean norm of $b$. For any $p$-dimensional vector of 
functions $h(x)$, let $\|h\|_{\infty} \equiv\left\|\left(\left\|h_{1}\right\|_{\text {sup }}, \ldots,\left\|h_{p}\right\|_{\text {sup }}\right)\right\|_{E}$ where $\left\|h_{j}\right\|_{\text {sup }} \equiv \sup \left\{\left|h_{j}(x)\right|\right.$ : $x \in \mathcal{X}\}$ and $h_{j}(x)$ denote the $j$ th component of $h$. Let $\widetilde{z}$ be the subvector of $z$ excluding the first component, say $z_{1}$ of $z$. Write $b_{1}=\left(b_{1,1}, \widetilde{b}_{1}\right)$ and $\beta_{1}=\left(\beta_{1,1}, \widetilde{\beta}_{1}\right)$. We assume the following regularity conditions.

Assumption 1. Assume that:

C1. $\Theta=\{-1,1\} \times \Upsilon$, where $\Upsilon$ is a compact subspace of $R^{k+p-1}$ and $\left(\widetilde{\beta}_{1}, \beta_{2}\right)$ is an interior point of $\Upsilon$.

C2. (a) The support of the distribution of $w$ is not contained in any proper linear subspace of $R^{k+p}$. (b) $0<P(d=1 \mid w)<1$ for almost every $w$. (c) For almost every $(\widetilde{z}, x)$, the distribution of $z_{1}$ conditional on $(\widetilde{z}, x)$ has everywhere positive density with respect to Lebesgue measure.

C3. $\operatorname{Med}(\varepsilon \mid z, x)=0$ for almost every $(z, x)$.

C4. There is a positive constant $L<\infty$ such that $\left|F\left(t_{1} ; b\right)-F\left(t_{2} ; b\right)\right| \leq L\left|t_{1}-t_{2}\right|$ for all $\left(t_{1}, t_{2}\right) \in R^{2}$ uniformly over $b \in \Theta$.

C5. $\|\widehat{G}-G\|_{\infty}=o_{p}(1)$.

Because the scale of $\beta$ for the model characterized by (2.4) cannot be identified, Assumption $\mathrm{C} 1$ imposes scale normalization by requiring that the absolute value of the first coefficient is unity. Assumption $\mathrm{C} 2$ implies that $F(t ; b)$ is absolutely continuous and has density $f(t ; b)$ for each $b \in\{-1,1\} \times \Upsilon$. Assumptions C1 - C3 are standard in the maximum score estimation literature (see e.g., Manski (1985), Horowitz (1992), and Florios and Skouras (2008)). Assumption C4 is a mild condition on the distribution of the index variable $w^{\prime} b$. Assumption C5 requires uniform consistency of the first stage estimation. This assumption can be easily verified for standard nonparametric estimators such as series estimators (Newey (1997, Theorem 1)) and the kernel regression estimator (Bierens (1983, Theorem 1), Bierens (1987, Theorem 2.3.1) and Andrews (1995, Theorem 1)).

Given these regularity conditions, we have the following result. 
Theorem 1 (Consistency). Let Assumption 1 (C1 - C5) hold. Then the two-stage estimator given by (2.6) converges to $\beta$ in probability as $N \longrightarrow \infty$.

In addition to consistency, we also study rate of convergence of the estimator $\widehat{\beta}$. Let $\widetilde{w} \equiv(\widetilde{z}, G(x)), \widetilde{b} \equiv\left(\widetilde{b}_{1}, b_{2}\right)$ and $\widetilde{\beta} \equiv\left(\widetilde{\beta}_{1}, \beta_{2}\right)$. Let $F_{\varepsilon}(\cdot \mid z, x)$ denote the distribution function of $\varepsilon$ conditional on $(z, x)$ and $g_{1}\left(z_{1} \mid \widetilde{z}, x\right)$ denote the density function of $z_{1}$ conditional on $(\widetilde{z}, x)$. Let $p_{1}(\cdot, \widetilde{z}, x)$ denote the partial derivative of $P(d=1 \mid z, x)$ with respect to $z_{1}$. Define the following matrix

$$
V \equiv \beta_{1,1} E\left[\tau p_{1}\left(-\widetilde{w}^{\prime} \widetilde{\beta} / \beta_{1,1}, \widetilde{z}, x\right) g_{1}\left(-\widetilde{w}^{\prime} \widetilde{\beta} / \beta_{1,1} \mid \widetilde{z}, x\right) \widetilde{w} \widetilde{w}^{\prime}\right] .
$$

Since the objective function of (2.5) is non-smooth, we require the nonparametric parameter of the estimation problem should possess certain degree of smoothness to facilitate derivation of the rate of convergence result. In particular, we consider the following well known class of smooth functions (see, e.g., van der Vaart and Wellner (1996, Section 2.7.1)) : for $0<\alpha<\infty$, let $C_{M}^{\alpha}$ denote the class of functions $f: \mathcal{X} \longmapsto \mathcal{R}$ with $\|f\|_{\alpha} \leq M$ where for any $q$ dimensional vector of non-negative integers $k=\left(k_{1}, \ldots, k_{q}\right)$,

$$
\|f\|_{\alpha} \equiv \max _{\sigma(k) \leq \underline{\alpha}}\left\|D^{k} f\right\|_{\text {sup }}+\max _{\sigma(k) \leq \underline{\alpha}} \sup _{x \neq x^{\prime}} \frac{\left|D^{k} f(x)-D^{k} f\left(x^{\prime}\right)\right|}{\left\|x-x^{\prime}\right\|_{E}^{\alpha-\underline{\alpha}}}
$$

where $\sigma(k) \equiv \sum_{j=1}^{q} k_{q}, \underline{\alpha}$ denotes the greatest integer smaller than $\alpha$, and $D^{k}$ is the differential operator

$$
D^{k} \equiv \frac{\partial^{\sigma(k)}}{\partial x_{1}^{k_{1}} \cdots \partial x_{q}^{k_{q}}} .
$$

Given the norm $\|\cdot\|_{\alpha}$, for any $p$-dimensional vector of functions $h(x)$, let $\|h\|_{\alpha, p} \equiv$ $\left\|\left(\left\|h_{1}\right\|_{\alpha}, \ldots,\left\|h_{p}\right\|_{\alpha}\right)\right\|_{E}$ where $h_{j}(x)$ denote the $j$ th component of $h$. Note that $\|\cdot\|_{\alpha, p}$ is a stronger norm than $\|\cdot\|_{\infty}$ used in condition C5 for the uniform consistency of the first stage estimator.

The regularity conditions imposed for the convergence rate result are stated as follows. 
Assumption 2. Assume that:

C6. The support of $\widetilde{z}$ is bounded.

C7. There is a positive constant $\bar{B}<\infty$ such that (i) for every $z_{1}$ and for almost $\operatorname{every}(\widetilde{z}, x)$,

$$
g_{1}\left(z_{1} \mid \widetilde{z}, x\right)<\bar{B},\left|\partial g_{1}\left(z_{1} \mid \widetilde{z}, x\right) / \partial z_{1}\right|<\bar{B}, \text { and }\left|\partial^{2} g_{1}\left(z_{1} \mid \widetilde{z}, x\right) / \partial z_{1}^{2}\right|<\bar{B}
$$

and (ii) for non-negative integers $i$ and $j$ satisfying $i+j \leq 2$,

$$
\left|\partial^{i+j} F_{\varepsilon}(t \mid z, x) / \partial t^{i} \partial z_{1}^{j}\right|<\bar{B}
$$

for every $t$ and $z_{1}$ and for almost every $(\widetilde{z}, x)$.

C8. All elements of the vector $\widetilde{w}$ have finite third absolute moments.

C9. The matrix $V$ is positive definite.

C10. For each $j \in\{1, \ldots, p\}, \Lambda_{j}=C_{M}^{\alpha}$ for some $\alpha>q$ and $M<\infty$.

C11. $\|\widehat{G}-G\|_{\alpha, p}=O_{p}\left(\varepsilon_{N}\right)$ where $\varepsilon_{N}$ is a non-stochastic positive real sequence such that $N^{1 / 3} \varepsilon_{N} \leq 1$ for each $N$.

Assumption C6 is standard in deriving asymptotic properties of Manski's maximum score estimator (see, e.g. Kim and Pollard (1990), pp. 213 - 216). Assumption C7 requires some smoothness of the density $g_{1}\left(z_{1} \mid \widetilde{z}, x\right)$ and the distribution $F_{\varepsilon}(t \mid z, x)$. Assumption C8 is mild. Since $-V$ corresponds to the second order derivative of $E\left[S_{N}(b, \gamma)\right]$ with respect to $\widetilde{b}$ evaluated at true parameter values, Assumption C9 is analogous to the classic condition of Hessian matrix being non-singular in the Mestimation framework. Assumption C10 imposes smoothness for the nonparametric parameter $\gamma$ and hence helps to control complexity of the space $\Lambda$. The requirement $\alpha>q$ is in line with the literature of two-stage semiparametric estimation with nonsmooth objective functions (See, e.g., Chen et.al. (2003, Example 2, pp. 1601-1603) and Ichimura and Lee (2010, Section 4.1, pp. 258-259)). 
Assumption C11 requires that the first stage estimator should converge under the norm $\|\cdot\|_{\alpha, p}$ at a rate no slower than $N^{-1 / 3}$. Note that convergence of $\widehat{G}$ to $G$ in the norm $\|\cdot\|_{\alpha, p}$ also implies uniform convergence of derivatives of $\widehat{G}$ to those of $G$. For integer-valued $\alpha>0$, Assumption C11 is fulfilled provided that for vector of non-negative integers $k=\left(k_{1}, \ldots, k_{q}\right)$ that satisfies $\sigma(k) \leq \alpha$,

$$
\left\|D^{k} \widehat{G}_{t, j}-D^{k} G_{t, j}\right\|_{\text {sup }}=O_{p}\left(\varepsilon_{N}\right)
$$

where $\widehat{G}_{t, j}(x)$ denotes the estimate of $G_{t, j}(x) \equiv E\left(y_{j} \mid x, d=t\right)$ for $(t, j) \in\{0,1\} \times$ $\{1, \ldots, p\}$. The condition (3.1) can also be verified for series estimators (Newey (1997, Theorem 1)) and the kernel regression estimator (Andrews (1995, Theorem 1)).

Theorem 2 (Rate of Convergence). In addition to Assumption 1 (C1 - C5), let Assumption 圆 $\boldsymbol{C}$ - C11) also hold. Then $\|\widehat{\beta}-\beta\|_{E}=O_{p}\left(N^{-1 / 3}\right)$.

If $G$ were priorly known to the researcher, the preference parameters $\beta$ could be estimated by the single stage maximum score estimator $\widehat{\beta}_{G}$, defined as

$$
\widehat{\beta}_{G}=\arg \max _{b \in \Theta} \mathrm{S}_{N}(b, G) .
$$

Kim and Pollard (1990) showed that $\widehat{\beta}_{G}$ converges to $\beta$ at cube root rate and established its asymptotic distribution. In the case of unknown $G$, Theorem 2 implies that the two-stage estimator $\widehat{\beta}$ retains the same convergence rate as the infeasible estimator $\widehat{\beta}_{G}$. Indeed if condition C11 is strengthened for faster convergence of first stage estimates, we can establish the oracle property that $N^{1 / 3}(\widehat{\beta}-\beta)$ and $N^{1 / 3}\left(\widehat{\beta}_{G}-\beta\right)$ have the same limiting distribution. Therefore, the inference on $\beta$ can be carried out by subsampling (Delgado et al. (2001)) since the standard bootstrap cannot be used to estimate the distribution of the maximum score estimator consistently (Abrevaya and Huang (2005)). We now state the asymptotic distributional equivalence result in the next theorem.

Theorem 3 (Asymptotic Distribution). Suppose all assumptions stated in Theorem 圆 hold with the additional restriction that the sequence $\varepsilon_{N}$ stated in $\boldsymbol{C 1 1}$ further 
satisfies $\varepsilon_{N}=o\left(N^{-1 / 3}\right)$. Then $N^{1 / 3}(\widehat{\beta}-\beta)$ is asymptotically equivalent in distribution to $N^{1 / 3}\left(\widehat{\beta}_{G}-\beta\right)$.

\section{Monte Carlo Simulations}

We employ the following data generating process (DGP) in simulation study of the two-stage maximum score estimator:

$$
d=1\left\{z \beta_{1}+G(x) \beta_{2}>\varepsilon\right\}
$$

where $G(x)=E(y \mid x, d=1)-E(y \mid x, d=0), z \sim$ Logistic, $x \sim N(0,1)$ and $\varepsilon=$ $0.25 \eta \sqrt{1+z^{2}+x^{2}}$ with $\eta \mid(x, z) \sim N(0,1)$. The scalar random variable $y$ is generated according to

$$
y=d\left(\gamma_{01}+\gamma_{11} m(x)+u_{1}\right)+(1-d)\left(\gamma_{00}+\gamma_{10} m(x)+u_{0}\right)
$$

where $\left(u_{1}, u_{0}\right)$ are independent of $(x, z, \varepsilon)$ and are jointly normally distributed with $E\left(u_{1}\right)=E\left(u_{0}\right)=0, \operatorname{Var}\left(u_{1}\right)=\operatorname{Var}\left(u_{0}\right)=\sigma_{u}^{2}$, and $\operatorname{Cov}\left(u_{1}, u_{0}\right)=\rho$. Given (4.1),

$$
G(x)=\gamma_{01}-\gamma_{00}+\left(\gamma_{11}-\gamma_{10}\right) m(x)
$$

We consider the following two types of the $m(x)$ function:

$$
\begin{aligned}
\text { Linear : } m(x) & =x, \\
\text { Nonlinear : } m(x) & =x^{2} \tan ^{-1} x .
\end{aligned}
$$

The true parameter values are specified as follows: $\beta_{1}=1, \beta_{2}=1, \gamma_{01}=0.2$, $\gamma_{11}=0.1, \gamma_{00}=0.1, \gamma_{10}=0.4, \rho=-0.8$, and $\sigma_{u}=0.33$.

We compare infeasible single-stage estimator using $(z, G(x))$ as regressors and also the feasible two-stage estimator using $(z, \widehat{G}(x))$ as regressors. We consider both parametric and nonparametric first stage estimators. For the former, we estimate $E(y \mid x, d=j)$ by running OLS of $y$ on $x$ with an intercept term using $d=j$ subsam- 
ples. For the latter, we implement Nadaraya-Watson kernel regression estimators. The nonparametric estimators of $E(y \mid x, d=j), j \in\{0,1\}$ are constructed as

$$
\frac{\sum_{i=1}^{N} y_{i} K\left(\widehat{\sigma}_{j}^{-1} h_{N}^{-1}\left(x-x_{i}\right)\right) 1\left\{d_{i}=j\right\}}{\sum_{i=1}^{N} K\left(\widehat{\sigma}_{j}^{-1} h_{N}^{-1}\left(x-x_{i}\right)\right) 1\left\{d_{i}=j\right\}}
$$

where $\widehat{\sigma}_{j}$ is the estimated standard deviation of $x_{i}$ conditional on $d_{i}=j, K($.$) is a$ univariate kernel function and $h_{N}$ is a deterministic bandwidth sequence. We use two types of kernel and bandwidth configurations.

For the first type, we use the second-order Gaussian kernel and set $h_{N}$ to be $c N^{-1 / 5}$ for various values of the bandwidth scale $c$. For the second type, we use the following 8th order kernel function (see, e.g., Bierens (1987, p. 112) and Andrews (1995, p. 567)):

$$
K(x) \equiv \sum_{s=1}^{4} a_{s}\left|b_{s}\right|^{-1} \exp \left[-x^{2} /\left(2 b_{s}^{2}\right)\right],
$$

where the constants $\left(a_{s}, b_{s}\right), s \in\{1, \ldots, 4\}$ satisfy

$$
\sum_{s=1}^{4} a_{s}=1 \text { and } \sum_{s=1}^{4} a_{s} b_{s}^{2 l}=0 \text { for } l \in\{1,2,3\} \text {. }
$$

We specify $b_{s}=s^{-1 / 2}$ and then solve $a_{s}$ as solution of the system of linear equations (4.6). Associated with this kernel, the bandwidth $h_{N}$ is set to be $c N^{-19 / 360}$ for various values of the scale $c 1$ By Theorem 1(b) of Andrews (1995), kernel regression estimator of $G(x)$ based on the second type configuration has convergence property required in (3.1) with $\sigma(k) \leq 2$ and $\varepsilon_{N}=N^{-41 / 120}$, thus fulfilling regularity conditions C5 and C11 of Section 3. The first stage estimation with the second-order kernels satisfies condition C5 but may not satisfy C11; however, we experiment with

\footnotetext{
${ }^{1}$ As noted by Bierens (1987, p. 113), choice of the constants $\left(a_{s}, b_{s}\right)$ for the kernel function is less crucial since its effect on asymptotic variance of the conditional mean estimator can be captured via the bandwidth scale $c$.
} 
the second-order kernels as well since kernel estimates with the second-order kernels often outperform those with the higher-order kernels in small samples 2

To implement the second-stage estimator using nonparametric first stage estimators, we trim the data by setting $\tau_{i}=1\left\{\left|x_{i}\right| \leq 1.95\right\}$ where $\tau_{i}$ is the weight introduced in (2.5). The estimates of $\beta_{1}$ and $\beta_{2}$ are obtained using grid search method. We report simulation results of $\widehat{\beta}_{2}$ for the parameter capturing the agent's uncertainty. Let $\widehat{\beta}_{2, \text { Single }}, \widehat{\beta}_{2, O L S}, \widehat{\beta}_{2, \text { Kernel_2nd }}$ and $\widehat{\beta}_{2, \text { Kernel_8th }}$ respectively denote the estimators $\widehat{\beta}_{2}$ that are constructed based on the infeasible single-stage, two-stage (OLS first stage) and two-stage (kernel regression first stage implemented with the 2nd and 8th order kernels) maximum score estimators. We compute bias, median, root mean squared error (RMSE), mean absolute deviation (mean AD) and median absolute deviation (median AD) of these estimators based on 1000 simulation repetitions for sample size $N \in\{300,500,1000\}$.

Tables 1-6 present simulation results for the four types of estimators of $\beta_{2}$ under linear and nonlinear designs of the $G(x)$ function. Tables 7 and 8 graph the simulated empirical distribution functions (edf) for $N^{1 / 3}\left(\widehat{\beta}_{2, \text { Single }}-\beta_{2}\right), N^{1 / 3}\left(\widehat{\beta}_{2, O L S}-\beta_{2}\right)$, $N^{1 / 3}\left(\widehat{\beta}_{2, \text { Kernel_2nd }}-\beta_{2}\right)$ and $N^{1 / 3}\left(\widehat{\beta}_{2, \text { Kernel_8th }}-\beta_{2}\right)$. As expected, for linear setup of $G$ the estimator $\widehat{\beta}_{2, O L S}$ enjoys the best overall finite-sample performance among all two-stage estimators. However, this estimator also incurs huge bias when agent's conditional expectation is nonlinear. For the estimators $\widehat{\beta}_{2, \text { Kernel_2nd }}$ and $\widehat{\beta}_{2, \text { Kernel_sth }}$, the function $G$ is nonparametrically estimated at the first stage. Hence regardless of nonlinearity of $G$, we see that the simulated bias, RMSE, mean AD and median AD of these estimators generally decrease as sample size grows.

We note that the edf curves of Tables 7 and 8 for the (kernel first-stage) two-stage estimators broadly match shapes of those for the infeasible estimators. Interestingly, finite sample behavior of the estimator $\widehat{\beta}_{2, \text { Kernel_2nd }}$ fits that of $\widehat{\beta}_{2, \text { Single }}$ better than its counterpart implemented with the 8th order kernel. Use of higher order kernels allows for verification of convergence of $\widehat{G}$ to $G$ in the strong norm $\|\cdot\|_{\alpha, p}$. However,

\footnotetext{
${ }^{2}$ See e.g., Marron and Wand (1992) and Efromovich (2001) for theoretical arguments why the higher-order kernels may perform poorly in small samples.
} 
as well known in the literature, the estimates with the higher-order kernels seem to perform poorly in simulations relative to those with the second-order kernels. The superb performance of $\widehat{\beta}_{2, \text { Kernel_2nd }}$ suggests that the asymptotic distributional equivalence result in Theorem 3 may not give us sharp asymptotics and there is scope to develop further asymptotic theory. This is an interesting future research topic.

\section{$5 \quad$ Further Applications of Two-Step Maximum Score Estimation with First-Stage Nonparametric Es- timation}

Our paper has been motivated by estimation problem in the binary choice model under uncertainty. However, the resulting estimator has wider applicability than just this model. To further motivate our two-step estimation procedure, this section gives a couple of additional econometric models for which unknown parameters can be estimated by maximum score with nonparametrically generated regressors.

We first consider maximum score estimation of an incomplete information games. Aradillas-Lopez (2012) developed a two-step procedure for estimation of incomplete

information games with Nash equilibrium behavior. Equation (2) of Aradillas-Lopez (2012, p. 123) gives a description of players' behavior in a $2 \times 2$ game:

$$
\begin{aligned}
& Y_{1}=1\left\{X_{1}^{\prime} \beta_{1}+\Delta_{1} \operatorname{Pr}\left[Y_{2}=1 \mid X\right]-\zeta_{1} \geq 0\right\}, \\
& Y_{2}=1\left\{X_{2}^{\prime} \beta_{2}+\Delta_{2} \operatorname{Pr}\left[Y_{1}=1 \mid X\right]-\zeta_{2} \geq 0\right\},
\end{aligned}
$$

where $Y_{p} \in\{0,1\}$ is the binary action for player $p=1,2, X_{p}$ and $\zeta_{p}$ are observable and unobservable payoff covariates, $X \equiv\left(X_{1}^{\prime}, X_{2}^{\prime}\right)^{\prime}$, and $\left\{\left(\beta_{p}, \Delta_{p}\right): p=1,2\right\}$ are unknown parameters.

Aradillas-Lopez (2012, Assumption A0, p. 122) assumed that players' behavior corresponds to a Bayesian-Nash equilibrium with a degenerate selection mechanism. He further assumed that $\zeta_{1}$ and $\zeta_{2}$ are independent of each other, independent of $X$, 
and of the selection mechanism.

We can make the same assumptions as in Aradillas-Lopez (2012), with one exception. As in the previous section, we consider $\operatorname{Med}\left(\zeta_{p} \mid X\right)=0$ almost surely, instead of assuming the full independence between $\zeta_{p}$ and $X$, where $p=1,2$. Allowing for dependence between $\zeta_{p}$ and $X$ might be important in applications when we suspect possible interactions between observed covariates and unobserved components that affect players' payoffs. Then for each $p=1,2$, we can estimate $\left(\beta_{p}, \Delta_{p}\right)$ by running maximum score regression of $Y_{p}$ on $X_{p}$ and $G_{-p}(X) \equiv \operatorname{Pr}\left[Y_{-p}=1 \mid X\right]$ with the nonparametric first stage estimation of $G_{-p}(X)$. Therefore, methodology of the present paper can be applied to extension of Aradillas-Lopez (2012)'s context allowing unobserved payoffs to exhibit unknown form of heteroskedasticity.

Our second application, which is based on Fox (2007), is maximum score estimation of multinomial discrete-choice models using a subset of choices under endogeneity. Fox (2007) proposed pairwise maximum score estimation of multinomial discrete-choice models using a subset of choices. For simplicity, assume that a researcher has data on only two choice, say 1 and 2 , among $J(\geq 3)$ alternatives, and also assume that there exists an endogenous covariate. Fox (2007, p. 1013) solved the endogeneity problem by including, instead of the endogenous covariate, fitted values from the OLS regression of the endogenous covariate, say price, on a vector of instruments. We can extend Fox (2007) to allow for nonparametric fitted values. Then this extension again can be accommodated in the framework of maximum score estimation with nonparametrically generated regressors.

\section{Conclusions}

This paper has developed maximum score estimation of preference parameters in the binary choice model under uncertainty in which the decision rule is affected by conditional expectations. The estimation procedure is implemented in two stages: we estimate conditional expectations nonparametrically in the first stage and obtain the maximum score estimate of the preference parameters in the second stage 
using choice data and the first stage estimates. The paper has shown consistency and convergence rate of the two-stage maximum score estimator. Moreover, we also establish the oracle property in terms of asymptotic equivalence in distribution of the two-stage estimator and its corresponding infeasible single-stage version. These results are of independent interest for maximum score estimation with nonparametrically generated regressors.

It would be an alternative approach to develop the second stage estimator using Horowitz (1992)'s smoothed maximum score estimator or using a Laplace estimator proposed in Jun, Pinkse, and Wan (2013). These alternative methods would produce faster convergence rates but require extra tuning parameters. Alternatively, we might build the second stage estimator based on Lewbel (2000), who introduced the idea of a special regressor satisfying certain conditional independence restriction. These are interesting future research topics.

\section{A Proof of Consistency}

Recall that $w=(z, G(x))$ and $S_{N}(b, \gamma)$ is the sample score function defined by (2.5). We first state and prove a preliminary lemma that will be invoked in proving Theorem 1 of the paper.

Lemma 1. Under Assumptions C1, C4 and C5,

$$
\sup _{b \in \Theta}\left|S_{N}(b, \widehat{G})-S_{N}(b, G)\right| \stackrel{p}{\longrightarrow} 0
$$

Proof of Lemma 1. Note that

$$
\left|S_{N}(b, \widehat{G})-S_{N}(b, G)\right| \leq \frac{1}{N} \sum_{i=1}^{N} \tau_{i} 1\left\{\left|\left(\widehat{G}\left(x_{i}\right)-G\left(x_{i}\right)\right)^{\prime} b_{2}\right| \geq\left|w_{i}^{\prime} b\right|\right\} .
$$

By Assumption $\mathrm{C} 1,\left\|b_{2}\right\|_{E}<B_{2}$ for some finite positive constant $B_{2}$. Therefore, the 
right-hand side of the inequality (A.2) is bounded above by

$$
\tilde{\Gamma}_{N} \equiv P_{N}\left(\tau=1, B_{2}\|\widehat{G}-G\|_{\infty} \geq\left|w^{\prime} b\right|\right)
$$

where $P_{N}$ denotes the empirical probability. Note that the term (A.3) is further bounded above by

$$
\Gamma_{N} \equiv P_{N}\left(B_{2}\|\widehat{G}-G\|_{\infty} \geq\left|w^{\prime} b\right|\right)
$$

Let $E_{\eta}$ denote the event $\|\widehat{G}-G\|_{\infty}<\eta$ for some $\eta>0$. Then given $\epsilon>0$,

$$
\begin{aligned}
& P\left(\sup _{b \in \Theta} \Gamma_{N}>\epsilon\right) \leq P\left(\sup _{b \in \Theta} \Gamma_{N}>\epsilon, E_{\eta}\right)+P\left(E_{\eta}^{c}\right) \\
\leq & P\left[\sup _{b \in \Theta} P_{N}\left(B_{2} \eta \geq\left|w^{\prime} b\right|\right)>\epsilon\right]+P\left(E_{\eta}^{c}\right) .
\end{aligned}
$$

By Assumption C5, $P\left(E_{\eta}^{c}\right) \longrightarrow 0$ as $N \longrightarrow \infty$. Hence, to show (A.1), it remains to establish that as $N \longrightarrow \infty$,

$$
P\left[\sup _{b \in \Theta} P_{N}\left(B_{2} \eta \geq\left|w^{\prime} b\right|\right)>\epsilon\right] \longrightarrow 0
$$

Note that by Assumption C4, $P\left(B_{2} \eta \geq\left|w^{\prime} b\right|\right) \leq 2 L B_{2} \eta$. Therefore, we have that

$$
\begin{aligned}
& P\left[\sup _{b \in \Theta} P_{N}\left(B_{2} \eta \geq\left|w^{\prime} b\right|\right)>\epsilon\right] \\
\leq & P\left[\sup _{b \in \Theta}\left|P_{N}\left(B_{2} \eta \geq\left|w^{\prime} b\right|\right)-P\left(B_{2} \eta \geq\left|w^{\prime} b\right|\right)\right|>\epsilon-2 L B_{2} \eta\right],
\end{aligned}
$$

where $\eta$ is taken to be sufficiently small such that $\epsilon-2 L B_{2} \eta>0$ for the given $\epsilon$. By Lemma 9.6, 9.7 (ii) and 9.12 (i) of Kosorok (2008), the family of sets $\left\{B_{2} \eta \geq\left|w^{\prime} b\right|\right\}$ for $b \in \Theta$ forms a Vapnik-Červonenkis class. Therefore, by Glivenko-Cantelli Theorem (see, e.g. Theorem 2.4.3 of van der Vaart and Wellner (1996)), the right-hand side of (A.6) tends to zero as $N \longrightarrow \infty$. Hence, the convergence result in (A.5) holds and Lemma 1 thus follows.

We now prove Theorem 1 for consistency of $\widehat{\beta}$. 
Proof of Theorem 1. For any $(b, \gamma)$, define

$$
S(b, \gamma) \equiv E\left[\tau(2 d-1) 1\left\{z^{\prime} b_{1}+\gamma(x)^{\prime} b_{2}>0\right\}\right]
$$

Given Assumptions C1 - C3 and by Manski (1985, Lemma 3, p. 321), $\beta$ uniquely satisfies $\beta=\arg \max _{b \in \Theta} S(b, G)$. We now look at the difference

$$
\left|S_{N}(b, \widehat{G})-S(b, G)\right| \leq\left|S_{N}(b, \widehat{G})-S_{N}(b, G)\right|+\left|S_{N}(b, G)-S(b, G)\right|,
$$

where by Lemma 1, the first term of the right-hand side of (A.7) converges to zero in probability uniformly over $b \in \Theta$, whilst by Manski (1985, Lemma 4, p. 321), the second term converges to zero almost surely uniformly over $b \in \Theta$. Therefore, we have that

$$
\sup _{b \in \Theta}\left|S_{N}(b, \widehat{G})-S(b, G)\right| \stackrel{p}{\longrightarrow} 0 .
$$

By Lemma 5 of Manski $(1985$, p. 322), $S(b, G)$ is continuous in $b$. Given these results, Theorem 1 thus follows by application of the consistency theorem in Newey and McFadden (1994, Theorem 2.1).

\section{B Lemma on the Rates of Convergence of a Two- Stage M-Estimator with a Non-smooth Crite- rion Function}

We first present and prove a general lemma establishing the rates of convergence of a general two-stage M-estimator under high level assumptions. In next section, we prove Theorem 2 by verifying these assumptions for the particular estimator given by (2.6) under the regularity conditions of $\mathrm{C} 1$ - C11.

To present a general result, let $s \mapsto m_{\theta, h}(s)$ be measurable functions indexed by parameters $(\theta, h)$. Let $\Theta$ and $H$ be the space of parameters $\theta$ and $h$, respectively. Let $\left(\theta^{*}, h^{*}\right)$ denote the true parameter value. We assume $\left(\theta^{*}, h^{*}\right) \in \Theta \times H$. Let $S_{N}(\theta, h) \equiv \sum_{i=1}^{N} m_{\theta, h}\left(s_{i}\right) / N$ be the empirical criterion of the M-estimation prob- 
lem where $\left(s_{i}\right)_{i=1}^{N}$ are i.i.d. random vectors. Suppressing the individual index, let $S(\theta, h) \equiv E\left[m_{\theta, h}(s)\right]$ be the population criterion. For a given first stage estimate $\widehat{h}$, let the estimator $\widehat{\theta}$ be constructed as

$$
\widehat{\theta}=\arg \sup _{\theta \in \Theta} S_{N}(\theta, \widehat{h})
$$

Let $d_{\Theta}\left(\theta, \theta^{*}\right)$ and $d_{H}\left(h, h^{*}\right)$ be non-negative functions measuring discrepancies between $\theta$ and $\theta^{*}$, and $h$ and $h^{*}$, respectively. Note that $d_{\Theta}$ and $d_{H}$ are usually related to but not necessarily the same as the metrics specified for the spaces $\Theta$ and $H$. Given a non-stochastic positive real sequence $\varepsilon_{N}$, define $H_{N}(C) \equiv\{h \in H$ : $\left.d_{H}\left(h, h^{*}\right) \leq C \varepsilon_{N}\right\}$. To simplify the presentation, we use the notation $\lesssim$ to denote being bounded above up to a universal constant. Define the recentered criterion

$$
\widetilde{S}_{N}(\theta, h) \equiv\left(S_{N}(\theta, h)-S_{N}\left(\theta^{*}, h\right)\right)-\left(S(\theta, h)-S\left(\theta^{*}, h\right)\right) .
$$

The following lemma modifies the rate of convergence results developed by van der Vaart (1998, Theorem 5.55) and provides sufficient conditions ensuring that $\hat{\theta}$ retains the same convergence rate as it would have if $h^{*}$ were known.

Lemma 2 (Rate of convergence for a general two-stage M-estimator). For any fixed and sufficiently large $C>0$, assume that for all sufficiently large $N$,

$$
\sup _{h \in H_{N}(C)}\left|S\left(\theta^{*}, h\right)-S\left(\theta^{*}, h^{*}\right)\right| \lesssim\left(C \varepsilon_{N}\right)^{2}
$$

and there is a sequence of non-stochastic functions $e_{N}: \Theta \times H_{N}(C) \longmapsto R$ such that for all sufficiently small $\delta>0$ and for every $(\theta, h) \in \Theta \times H_{N}(C)$ satisfying $d_{\Theta}\left(\theta, \theta^{*}\right) \leq \delta$,

$$
\begin{aligned}
& S(\theta, h)-S\left(\theta^{*}, h^{*}\right)+e_{N}(\theta, h) \lesssim-d_{\Theta}^{2}\left(\theta, \theta^{*}\right)+d_{H}^{2}\left(h, h^{*}\right), \\
& \sup _{d_{\Theta}\left(\theta, \theta^{*}\right) \leq \delta,(\theta, h) \in \Theta \times H_{N}(C)}\left|e_{N}(\theta, h)\right| \lesssim C \delta \varepsilon_{N},
\end{aligned}
$$


and

$$
E\left[\sup _{d_{\Theta}\left(\theta, \theta^{*}\right) \leq \delta,(\theta, h) \in \Theta \times H_{N}(C)}\left|\widetilde{S}_{N}(\theta, h)\right|\right] \lesssim \frac{\phi_{N}(\delta)}{\sqrt{N}},
$$

where $\phi_{N}(\delta)$ is a sequence of functions defined on $(0, \infty)$ and satisfies that $\phi_{N}(\delta) \delta^{-\alpha}$ is decreasing for some $\alpha<2$. Suppose $d_{H}\left(\widehat{h}, h^{*}\right)=O_{p}\left(\varepsilon_{N}\right), d_{\Theta}\left(\widehat{\theta}, \theta^{*}\right)=o_{p}(1)$ and there is a non-stochastic positive real sequence $\delta_{N}$ which tends to zero as $N \longrightarrow \infty$ and satisfies that $\varepsilon_{N} \leq \delta_{N}$ and $\phi_{N}\left(\delta_{N}\right) \leq \sqrt{N} \delta_{N}^{2}$ for every $N$. Then $d_{\Theta}\left(\widehat{\theta}, \theta^{*}\right)=$ $O_{p}\left(\delta_{N}\right)$.

Proof. Based on the peeling technique of van der Vaart (1998, Theorem 5.55), for each natural number $N$, integer $j$ and positive real $M$, construct the set

$A_{N, j, M}(C) \equiv\left\{(\theta, h) \in \Theta \times H_{N}(C): 2^{j-1} \delta_{N}<d_{\Theta}\left(\theta, \theta^{*}\right) \leq 2^{j} \delta_{N}, d_{H}\left(h, h^{*}\right) \leq 2^{-M} d_{\Theta}\left(\theta, \theta^{*}\right)\right\}$.

Then we have that for any $\epsilon>0$,

$$
\begin{aligned}
& P\left(d_{\Theta}\left(\widehat{\theta}, \theta^{*}\right) \geq 2^{M}\left(\delta_{N}+d_{H}\left(\widehat{h}, h^{*}\right)\right), \widehat{h} \in H_{N}(C)\right) \\
\leq & P\left(2 d_{\Theta}\left(\widehat{\theta}, \theta^{*}\right)>\epsilon\right)+P\left((\widehat{\theta}, \widehat{h}) \in \bigcup_{j \geq M, 2^{j} \delta_{N} \leq \epsilon} A_{N, j, M}(C)\right) \\
\leq & P\left(2 d_{\Theta}\left(\widehat{\theta}, \theta^{*}\right)>\epsilon\right)+ \\
& \sum_{j \geq M, 2^{j} \delta_{N} \leq \epsilon} P\left(\sup _{(\theta, h) \in A_{N, j, M}(C)}\left[S_{N}(\theta, h)-S_{N}\left(\theta^{*}, h\right)\right] \geq 0\right)
\end{aligned}
$$

where the last inequality follows from the definition of $\widehat{\theta}$ given by (B.1). Since $d_{\Theta}\left(\widehat{\theta}, \theta^{*}\right)=o_{p}(1)$, the term $P\left(2 d_{\Theta}\left(\widehat{\theta}, \theta^{*}\right)>\epsilon\right)$ tends to zero as $N \longrightarrow \infty$. Hence the remaining part of the proof is to bound the terms in the sum (B.7).

Let $N$ be large enough such that (B.3) holds and choose $\epsilon$ to be small enough such that assumptions (B.4), (B.5) and (B.6) hold for every $\delta \leq \epsilon$. Note that for every sufficiently large $M$, if $(\theta, h) \in A_{N, j, M}(C)$, then $d_{H}^{2}\left(h, h^{*}\right)-d_{\Theta}^{2}\left(\theta, \theta^{*}\right) \lesssim-\delta_{N}^{2} 2^{2 j}$ so that by (B.4),

$$
S(\theta, h)-S\left(\theta^{*}, h^{*}\right)+e_{N}(\theta, h) \lesssim-\delta_{N}^{2} 2^{2 j}
$$


and thus

$$
S_{N}(\theta, h)-S_{N}\left(\theta^{*}, h\right) \lesssim\left[\widetilde{S}_{N}(\theta, h)+S\left(\theta^{*}, h^{*}\right)-S\left(\theta^{*}, h\right)-e_{N}(\theta, h)\right]-\delta_{N}^{2} 2^{2 j}
$$

Therefore, by Markov inequality, each term in the sum (B.7) can be bounded above by

$$
\delta_{N}^{-2} 2^{-2 j} E\left[\sup _{(\theta, h) \in A_{N, j, M}(C)}\left|\widetilde{S}_{N}(\theta, h)+S\left(\theta^{*}, h^{*}\right)-S\left(\theta^{*}, h\right)-e_{N}(\theta, h)\right|\right] .
$$

By (B.3), (B.5), (B.6) and applying triangular inequality, the term (B.9) is bounded above by

$$
\delta_{N}^{-2} 2^{-2 j}\left[N^{-1 / 2} \phi_{N}\left(2^{j} \delta_{N}\right)+2^{j} C \delta_{N} \varepsilon_{N}+\left(C \varepsilon_{N}\right)^{2}\right]
$$

By the monotonicity property of the mapping $\delta \mapsto \phi_{N}(\delta) \delta^{-\alpha}$, we have that $\phi_{N}\left(2^{j} \delta_{N}\right) \leq$ $2^{j \alpha} \phi_{N}\left(\delta_{N}\right)$. Furthermore, since $\phi_{N}\left(\delta_{N}\right) \leq \sqrt{N} \delta_{N}^{2}$, the first term in the bracket of (B.10) can thus be bounded by $2^{j \alpha} \delta_{N}^{2}$. Given that $\varepsilon_{N} \leq \delta_{N}$, the term (B.10) can be further bounded above by $2^{j(\alpha-2)}+C 2^{-j}+C^{2} 2^{-2 j}$. Using this fact and the condition $\alpha<2$, it follows that the sum (B.7) tends to zero as $M \longrightarrow \infty$.

Since $d_{H}\left(\widehat{h}, h^{*}\right)=O_{p}\left(\varepsilon_{N}\right), P\left(\widehat{h} \in H_{N}(C)\right)$ can be made arbitrarily close to 1 by choosing a sufficiently large value of $C$ for every sufficiently large $N$. Therefore, Lemma2 follows by putting together all these results and noting that $\delta_{N}+d_{H}\left(\widehat{h}, h^{*}\right)=$ $O_{p}\left(\delta_{N}\right)$.

\section{Proof of the Rate of Convergence for $\widehat{\beta}$}

To establish the convergence rate of $\widehat{\beta}$, we apply Lemma 2 by setting $(\theta, h)=(b, \gamma)$, $\left(\theta^{*}, h^{*}\right)=(\beta, G), \Theta=\{-1,1\} \times \Upsilon, H=\Lambda, s=(\tau, d, z, x)$ and

$$
m_{b, \gamma}(s) \equiv \tau(2 d-1) 1\left\{z^{\prime} b_{1}+\gamma(x)^{\prime} b_{2}>0\right\}
$$


Assumptions (B.3), (B.4), (B.5) and (B.6) of Lemma 2 are non-trivial and will be verified using primitive conditions C1 - C11 of the model. Assumption (B.4 $)$ is concerned with the quadratic expansion of $S(b, \gamma)$ around $(\beta, G)$ by which we obtain the functional form of $e_{N}(b, \gamma)$. Recall that $w=(z, G(x)), z=\left(z_{1}, \widetilde{z}\right), \widetilde{w}=(\widetilde{z}, G(x))$, $b_{1}=\left(b_{1,1}, \widetilde{b}_{1}\right), \beta_{1}=\left(\beta_{1,1}, \widetilde{\beta}_{1}\right), \widetilde{b}=\left(\widetilde{b}_{1}, b_{2}\right)$ and $\widetilde{\beta}=\left(\widetilde{\beta}_{1}, \beta_{2}\right)$. The following lemma will be used to establish expansion of the population criterion $S(b, \gamma)$.

Lemma 3. Under conditions $C 3$ and $C 7$, the sign of $p_{1}\left(-\widetilde{w}^{\prime} \widetilde{\beta} / \beta_{1,1}, \widetilde{z}, x\right)$ is the same as that of $\beta_{1,1}$ for almost every $(\widetilde{z}, x)$.

Proof. Note that the model (2.2) implies that

$$
P(d=1 \mid z, x)=F_{\varepsilon}\left(w^{\prime} \beta \mid z, x\right) .
$$

Thus, by $\mathrm{C} 7(\mathrm{ii}), P(d=1 \mid z, x)$ is differentiable with respect to $z_{1}$ and

$$
\frac{\partial}{\partial z_{1}} P(d=1 \mid z, x)=\left.\beta_{1,1} \frac{\partial}{\partial t} F_{\varepsilon}(t \mid z, x)\right|_{t=w^{\prime} \beta}+\left.\frac{\partial}{\partial z_{1}} F_{\varepsilon}(t \mid z, x)\right|_{t=w^{\prime} \beta} .
$$

Consider the mapping $\left.z_{1} \mapsto h\left(z_{1}\right) \equiv \frac{\partial}{\partial z_{1}} F_{\varepsilon}(t \mid z, x)\right|_{t=z_{1} \beta_{1,1}+\widetilde{w}^{\prime} \widetilde{\beta}}$. By C3, $h\left(-\widetilde{w}^{\prime} \widetilde{\beta} / \beta_{1,1}\right)=$ 0 for almost every $(\widetilde{z}, x)$. Therefore, Lemma 3 follows from this fact and the monotonicity of $F_{\varepsilon}(t \mid z, x)$ in the argument $t$.

By assumption $\mathrm{C} 1$, the space of the coefficient $b_{1,1}$ is $\{-1,1\}$ and thus $b_{1,1}=\beta_{1,1}$ when $\|b-\beta\|_{E}<\delta$ for $\delta$ small enough. Let $p(z, x) \equiv P(d=1 \mid z, x)$ and

$$
S_{1}(\widetilde{b}, \gamma) \equiv E\left[\tau(2 p(z, x)-1) 1\left\{z_{1} \beta_{1,1}+\widetilde{z}^{\prime} \widetilde{b}_{1}+\gamma(x)^{\prime} b_{2}>0\right\}\right]
$$

We now derive the quadratic expansion of $S_{1}(\widetilde{b}, \gamma)$ around $(\widetilde{\beta}, G)$.

Lemma 4. For sufficiently small $\|\widetilde{b}-\widetilde{\beta}\|_{E}$ and $\|\gamma-G\|_{\infty}$ and under conditions $C 3$, C7, C8 and C9, we have that

$$
\left|S_{1}(\widetilde{\beta}, \gamma)-S_{1}(\widetilde{\beta}, G)\right| \lesssim\|\gamma-G\|_{\infty}^{2}
$$


and there are constants $c_{1}>0$ and $c_{2} \geq 0$ such that

$$
S_{1}(\widetilde{b}, \gamma)-S_{1}(\widetilde{\beta}, G)+e(\widetilde{b}, \gamma) \leq-c_{1}\|\widetilde{b}-\widetilde{\beta}\|_{E}^{2}+c_{2}\|\gamma-G\|_{\infty}^{2}
$$

for some function $e(\widetilde{b}, \gamma)$ that satisfies

$$
|e(\widetilde{b}, \gamma)| \lesssim\|\widetilde{b}-\widetilde{\beta}\|_{E}\|\gamma-G\|_{\infty} .
$$

Proof. We prove Lemma 4 explicitly for the case $\beta_{1,1}=1$. Proof for the case $\beta_{1,1}=$ -1 can be done by similar arguments.

Suppose now $\beta_{1,1}=1$. Then

$$
\begin{aligned}
& S_{1}(\widetilde{b}, \gamma)-S_{1}(\widetilde{\beta}, G) \\
= & E\left(\tau(2 p(z, x)-1)\left[1\left\{z_{1}+\widetilde{z} \widetilde{\beta}_{1}+G(x)^{\prime} \beta_{2} \leq 0\right\}-1\left\{z_{1}+\widetilde{z} \widetilde{b}_{1}+\gamma(x)^{\prime} b_{2} \leq 0\right\}\right]\right) .
\end{aligned}
$$

Let

$$
\begin{aligned}
\lambda(t) & \equiv \widetilde{z}^{\prime}\left(\widetilde{\beta}_{1}+t\left(\widetilde{b}_{1}-\widetilde{\beta}_{1}\right)\right)+(G(x)+t(\gamma(x)-G(x)))^{\prime}\left(\beta_{2}+t\left(b_{2}-\beta_{2}\right)\right), \\
\Psi(t) & \equiv-E\left(\tau(2 p(z, x)-1) 1\left\{z_{1}+\lambda(t) \leq 0\right\}\right) .
\end{aligned}
$$

The first-order and second-order derivatives of $\Psi(t)$ are derived as follows:

$$
\begin{aligned}
\Psi^{\prime}(t)= & E\left(\tau \lambda^{\prime}(t)(2 p(-\lambda(t), \widetilde{z}, x)-1) g_{1}(-\lambda(t) \mid \widetilde{z}, x)\right), \\
\Psi^{\prime \prime}(t)= & -E\left\{\tau ( \lambda ^ { \prime } ( t ) ) ^ { 2 } \left[2 p_{1}(-\lambda(t), \widetilde{z}, x) g_{1}(-\lambda(t) \mid \widetilde{z}, x)\right.\right. \\
& \left.\left.+(2 p(-\lambda(t), \widetilde{z}, x)-1) \frac{\partial}{\partial z_{1}} g_{1}(-\lambda(t) \mid \widetilde{z}, x)\right]\right\} \\
& +E\left(2 \tau[(2 p(-\lambda(t), \widetilde{z}, x)-1)] g_{1}(-\lambda(t) \mid \widetilde{z}, x)(\gamma(x)-G(x))^{\prime}\left(b_{2}-\beta_{2}\right)\right) .
\end{aligned}
$$

Then the second order expansion of $S_{1}(\widetilde{b}, \gamma)-S_{1}(\widetilde{\beta}, G)$ takes the form

$$
\Psi^{\prime}(0)+\Psi^{\prime \prime}(0) / 2+o\left(\left(\max \left\{\|\widetilde{b}-\widetilde{\beta}\|_{E},\|\gamma-G\|_{\infty}\right\}\right)^{2}\right)
$$


where by $\mathrm{C} 7$ and $\mathrm{C} 8$, the remainder term has the stated order uniformly over $\widetilde{b}$ and $\gamma$. Given assumption C3, it follows that $p\left(-\widetilde{w}^{\prime} \widetilde{\beta}, \widetilde{z}, x\right)=1 / 2$ for almost every $(\widetilde{z}, x)$. Let

$$
\kappa(\widetilde{z}, x)=2 p_{1}\left(-\widetilde{w}^{\prime} \widetilde{\beta}, \widetilde{z}, x\right) g_{1}\left(-\widetilde{w}^{\prime} \widetilde{\beta} \mid \widetilde{z}, x\right)
$$

Then we have that

$$
\begin{aligned}
\Psi^{\prime}(0)+\Psi^{\prime \prime}(0) / 2 & =-E\left(\tau \kappa(\widetilde{z}, x)\left(\widetilde{w}^{\prime}(\widetilde{b}-\widetilde{\beta})+(\gamma(x)-G(x))^{\prime} \beta_{2}\right)^{2}\right) \\
& =-\left(A_{1}+A_{2}+e(\widetilde{b}, \gamma)\right)
\end{aligned}
$$

where

$$
\begin{aligned}
A_{1}(\widetilde{b}) & \equiv(\widetilde{b}-\widetilde{\beta})^{\prime} E\left(\tau \kappa(\widetilde{z}, x) \widetilde{w} \widetilde{w}^{\prime}\right)(\widetilde{b}-\widetilde{\beta}), \\
A_{2}(\gamma) & \equiv E\left(\tau \kappa(\widetilde{z}, x)(\gamma(x)-G(x))^{\prime} \beta_{2} \beta_{2}^{\prime}(\gamma(x)-G(x))\right) \\
e(\widetilde{b}, \gamma) & \equiv 2(\widetilde{b}-\widetilde{\beta})^{\prime} E\left(\tau \kappa(\widetilde{z}, x) \widetilde{w} \beta_{2}^{\prime}(\gamma(x)-G(x))\right) .
\end{aligned}
$$

Under condition C9, $E\left(\tau \kappa(\widetilde{z}, x) \widetilde{w} \widetilde{w}^{\prime}\right)$ is positive definite, so that $A_{1} \geq c_{1}\|\widetilde{b}-\widetilde{\beta}\|_{E}^{2}$ for some positive real constant $c_{1}$. By Lemma [3, $p_{1}\left(-\widetilde{w}^{\prime} \widetilde{\beta}, \widetilde{z}, x\right) \geq 0$ and thus $\kappa(\widetilde{z}, x) \geq 0$. By Cauchy-Schwarz inequality, $0 \leq A_{2} \leq c_{2}\|\gamma-G\|_{\infty}^{2}$, where $c_{2} \equiv$ $E(\tau \kappa(\widetilde{z}, x))\left\|\beta_{2}\right\|_{E}^{2} \geq 0$, and the function $e(\widetilde{b}, \gamma)$ satisfies that

$$
\begin{aligned}
|e(\widetilde{b}, \gamma)| & \leq 2 E\left(\tau \kappa(\widetilde{z}, x)\left|(\widetilde{b}-\widetilde{\beta})^{\prime} \widetilde{w} \beta_{2}^{\prime}(\gamma(x)-G(x))\right|\right) \\
& \leq 2 E\left(\tau \kappa(\widetilde{z}, x)\|\widetilde{w}\|_{E}\right)\left\|\beta_{2}\right\|_{E}\|\widetilde{b}-\widetilde{\beta}\|_{E}\|\gamma-G\|_{\infty}
\end{aligned}
$$

Hence Lemma 4 follows by noting that when $\|\widetilde{b}-\widetilde{\beta}\|_{E}$ and $\|\gamma-G\|_{\infty}$ are sufficiently small,

$$
\left|S_{1}(\widetilde{\beta}, \gamma)-S_{1}(\widetilde{\beta}, G)\right|=\left|A_{2}+o\left(\|\gamma-G\|_{\infty}^{2}\right)\right| \leq c_{2}\|\gamma-G\|_{\infty}^{2}
$$


and

$$
\begin{aligned}
S_{1}(\widetilde{b}, \gamma)-S_{1}(\widetilde{\beta}, G)+e(\widetilde{b}, \gamma) & \leq-A_{1}+A_{2} \\
& \leq-c_{1}\|\widetilde{b}-\widetilde{\beta}\|_{E}^{2}+c_{2}\|\gamma-G\|_{\infty}^{2}
\end{aligned}
$$

We now verify assumption (B.6) of Lemma 2, Note that for $\delta$ sufficiently small, assumption C1 implies that $b_{1,1}=\beta_{1,1}$ when $\|b-\beta\|_{E} \leq \delta$. Therefore we can focus on analyzing (․․6) for the case of $b_{1,1}=\beta_{1,1}$ and $\|\widetilde{b}-\widetilde{\beta}\|_{E} \leq \delta$. For any $s=(\tau, d, z, x)$, consider the following recentered function

$\widetilde{m}_{\widetilde{b}, \gamma}(s) \equiv \tau(2 d-1)\left[1\left\{z_{1} \beta_{1,1}+\widetilde{z} \widetilde{b}_{1}+\gamma(x)^{\prime} b_{2}>0\right\}-1\left\{z_{1} \beta_{1,1}+\widetilde{z} \widetilde{\beta}_{1}+\gamma(x)^{\prime} \beta_{2}>0\right\}\right]$

and the class of functions

$$
\digamma_{\delta, \varepsilon} \equiv\left\{\widetilde{m}_{\widetilde{b}, \gamma}:\|\widetilde{b}-\widetilde{\beta}\|_{E} \leq \delta,\|\gamma-G\|_{\alpha, p} \leq \varepsilon\right\}
$$

Let $\|\cdot\|_{L_{r}(P)}$ denote the $L_{r}(P)$ norm such that $\|f\|_{L_{r}(P)} \equiv\left[E\left(|f(\tau, d, z, x)|^{r}\right)\right]^{1 / r}$ for any measurable function $f$. For any $\epsilon>0$, let $N_{[]}\left(\epsilon, \digamma, L_{r}(P)\right)$ denote the $L_{r}(P)$ - bracketing number for a given function space $\digamma$. Namely, $N_{[]}\left(\epsilon, \digamma, L_{r}(P)\right)$ is the minimum number of $L_{r}(P)$ - brackets of length $\epsilon$ required to cover $\digamma$ (see e.g., van der Vaart (1998, p. 270)). The logarithm of bracketing number for $\digamma$ is referred to as the bracketing entropy for $\digamma$. Assumption (B.6) is a stochastic equicontinuity condition concerning the complexity of the function space $\digamma_{\delta, \varepsilon}$ in terms of its envelope function and bracketing entropy. Let $M_{\delta, \varepsilon}$ denote an envelope for $\digamma_{\delta, \varepsilon}$ such that $\left|\widetilde{m}_{\tilde{b}, \gamma}(s)\right|$ $\leq\left|M_{\delta, \varepsilon}(s)\right|$ for all $s$ and for all $\widetilde{m}_{\widetilde{b}, \gamma} \in \digamma_{\delta, \varepsilon}$. The next lemma derives the envelope function $M_{\delta, \varepsilon}$.

Lemma 5. Let $\delta$ and $\varepsilon$ be sufficiently small. Then under conditions $C 1, C 4, C 6$ and 
C10, for some real constants $a_{1}>0$ and $a_{2}>0$, we can take

$$
M_{\delta, \varepsilon}=1\left\{a_{1} \max \{\delta, \varepsilon\} \geq\left|w^{\prime} \beta\right|\right\}
$$

and furthermore,

$$
\left\|M_{\delta, \varepsilon}\right\|_{L_{2}(P)} \leq a_{2} \sqrt{\max \{\delta, \varepsilon\}}
$$

Proof. Note that

$$
\begin{aligned}
& \left|\widetilde{m}_{\tilde{b}, \gamma}(\tau, d, z, x)\right| \\
& \leq 1\left\{z_{1} \beta_{1,1}+\widetilde{z} \widetilde{b}_{1}+\gamma(x)^{\prime} b_{2}>0 \geq z^{\prime} \beta_{1}+\gamma(x)^{\prime} \beta_{2}\right. \text { or } \\
& \left.z^{\prime} \beta_{1}+\gamma(x)^{\prime} \beta_{2}>0 \geq z_{1} \beta_{1,1}+\widetilde{z}^{\prime} \widetilde{b}_{1}+\gamma(x)^{\prime} b_{2}\right\} \text {. }
\end{aligned}
$$

Under condition C6, there is a positive real constant $B$ such that $\|\widetilde{z}\|_{E}<B$ with probability 1. Hence if $\|\widetilde{b}-\beta\|_{E} \leq \delta$ and $\|\gamma-G\|_{\alpha, p} \leq \varepsilon$, then we have that

$$
\begin{array}{ll} 
& z_{1} \beta_{1,1}+\widetilde{z}^{\prime} \widetilde{b}_{1}+\gamma(x)^{\prime} b_{2}>0 \geq z^{\prime} \beta_{1}+\gamma(x)^{\prime} \beta_{2} \\
\Longleftrightarrow & \widetilde{z}^{\prime}\left(\widetilde{b}_{1}-\widetilde{\beta}_{1}\right)+\gamma(x)^{\prime}\left(b_{2}-\beta_{2}\right)>-\left[z^{\prime} \beta_{1}+\gamma(x)^{\prime} \beta_{2}\right] \geq 0 \\
\Longrightarrow & \delta\left[\|\widetilde{z}\|_{E}+\|\gamma\|_{\infty}\right] \geq-\left[z^{\prime} \beta_{1}+\gamma(x)^{\prime} \beta_{2}\right] \text { and } 0 \geq w^{\prime} \beta+(\gamma(x)-G(x))^{\prime} \beta_{2} \\
\Longrightarrow & w^{\prime} \beta+(\gamma(x)-G(x))^{\prime} \beta_{2} \geq-\delta\left[\|\widetilde{z}\|_{E}+\varepsilon+\|G\|_{\infty}\right] \text { and } \varepsilon\left\|\beta_{2}\right\|_{E} \geq w^{\prime} \beta \\
\Longrightarrow & \delta\left[B+\varepsilon+\|G\|_{\infty}\right]+\varepsilon\left\|\beta_{2}\right\|_{E} \geq w^{\prime} \beta \geq-\delta\left[B+\varepsilon+\|G\|_{\infty}\right]-\varepsilon\left\|\beta_{2}\right\|_{E}
\end{array}
$$

Based on similar arguments, it also follows that

$$
\begin{aligned}
& z^{\prime} \beta_{1}+\gamma(x)^{\prime} \beta_{2}>0 \geq z_{1} \beta_{1,1}+\widetilde{z}^{\prime} \widetilde{b}_{1}+\gamma(x)^{\prime} b_{2} \\
\Longrightarrow & \delta\left[B+\varepsilon+\|G\|_{\infty}\right]+\varepsilon\left\|\beta_{2}\right\|_{E} \geq w^{\prime} \beta \geq-\delta\left[B+\varepsilon+\|G\|_{\infty}\right]-\varepsilon\left\|\beta_{2}\right\|_{E}
\end{aligned}
$$

Therefore, Lemma 5 follows by noting that for $\varepsilon$ sufficiently small (e.g., $\varepsilon<1$ ), we can take

$$
M_{\delta, \varepsilon}=1\left\{a_{1} \max \{\delta, \varepsilon\} \geq\left|w^{\prime} \beta\right|\right\}
$$

where $a_{1} \equiv 2 \max \left\{\left(B+1+\|G\|_{\infty}\right),\left\|\beta_{2}\right\|_{E}\right\}$. By C1 and C10, $0<a_{1}<\infty$ and 
hence by $\mathrm{C} 4,\left\|M_{\delta, \varepsilon}\right\|_{L_{2}(P)} \leq a_{2} \sqrt{\max \{\delta, \varepsilon\}}$ with $a_{2} \equiv \sqrt{2 a_{1} L}$ where $L$ is the positive constant stated in condition $\mathrm{C} 4$.

The following lemma establishes the bound for the bracketing entropy for $\digamma_{\delta, \varepsilon}$.

Lemma 6. Given conditions C1, C4, C6, C7, C8 and C10, we have that for sufficiently small $\delta$ and $\varepsilon$,

$$
\log N_{[]}\left(\epsilon, \digamma_{\delta, \varepsilon}, L_{2}(P)\right) \lesssim(\max \{\delta, \varepsilon\})^{q / \alpha} \epsilon^{-2 q / \alpha}
$$

Proof. For $j \in\{1, \ldots, p\}$, let $\widetilde{\Lambda}_{j}(\varepsilon)$ and $\widetilde{\Lambda}_{j} \mathbf{B}_{j}(\delta, \varepsilon)$ be classes of functions defined as

$$
\begin{aligned}
\widetilde{\Lambda}_{j}(\varepsilon) & \equiv\left\{\left(\gamma_{j}-G_{j}\right) / \varepsilon:\left\|\gamma_{j}-G_{j}\right\|_{\alpha} \leq \varepsilon\right\} \\
\widetilde{\Lambda}_{j} \mathbf{B}_{j}(\delta, \varepsilon) & \equiv\left\{\left(\gamma_{j}(x)-G_{j}(x)\right)\left(b_{2, j}-\beta_{2, j}\right) /(\varepsilon \delta):\left\|\gamma_{j}-G_{j}\right\|_{\alpha} \leq \varepsilon,\left|b_{2, j}-\beta_{2, j}\right| \leq \delta\right\}
\end{aligned}
$$

Assumption C10 implies that both $\widetilde{\Lambda}_{j}(\varepsilon)$ and $\widetilde{\Lambda}_{j} \mathbf{B}_{j}(\delta, \varepsilon)$ are $C_{1}^{\alpha}$. By Corollary 2.7.2 of van der Vaart and Wellner (1996, p. 157), we have that for $j \in\{1, \ldots, p\}$,

$$
\log N_{[]}\left(\epsilon^{2}, \widetilde{\Lambda}_{j}(\varepsilon), L_{1}(P)\right) \lesssim \epsilon^{-2 q / \alpha} \text { and } \log N_{[]}\left(\epsilon^{2}, \widetilde{\Lambda}_{j} \mathbf{B}_{j}(\delta, \varepsilon), L_{1}(P)\right) \lesssim \epsilon^{-2 q / \alpha}
$$

Note that for $s=(\tau, d, z, x), \widetilde{m}_{\widetilde{b}, \gamma}(s)$ defined by (C.5) can be rewritten as

$$
\widetilde{m}_{\widetilde{b}, \gamma}(s)=\tau d[1\{h(s ; \widetilde{b})>0\}-1\{h(s ; \widetilde{\beta})>0\}]+\tau(1-d)[1\{h(s ; \widetilde{b}) \leq 0\}-1\{h(s ; \widetilde{\beta}) \leq 0\}]
$$

where

$$
\begin{aligned}
h(s ; \widetilde{b}) & \equiv w^{\prime} \beta+\widetilde{w}^{\prime}(\widetilde{b}-\widetilde{\beta})+(\gamma(x)-G(x))^{\prime}\left(b_{2}-\beta_{2}\right)+(\gamma(x)-G(x))^{\prime} \beta_{2}, \\
h(s ; \widetilde{\beta}) & \equiv w^{\prime} \beta+(\gamma(x)-G(x))^{\prime} \beta_{2} .
\end{aligned}
$$


Consider the following spaces:

$$
\begin{aligned}
\Theta_{1} & \equiv\left\{\widetilde{w}^{\prime}(\widetilde{b}-\widetilde{\beta}):\|\widetilde{b}-\widetilde{\beta}\|_{E} \leq \delta\right\}, \\
\Theta_{2, j} & \equiv\left\{\left(\gamma_{j}(x)-G_{j}(x)\right)\left(b_{2, j}-\beta_{2, j}\right):\left\|\gamma_{j}-G_{j}\right\|_{\alpha} \leq \varepsilon,\left|b_{2, j}-\beta_{2, j}\right| \leq \delta\right\} \text { for } j \in\{1, \ldots, p\}, \\
\Theta_{2} & \equiv\left\{(\gamma(x)-G(x))^{\prime}\left(b_{2}-\beta_{2}\right):\|\gamma-G\|_{\alpha, p} \leq \varepsilon,\left\|b_{2}-\beta_{2}\right\|_{E} \leq \delta\right\}, \\
\Theta_{3, j} & \equiv\left\{\left(\gamma_{j}(x)-G_{j}(x)\right) \beta_{2, j}:\left\|\gamma_{j}-G_{j}\right\|_{\alpha} \leq \varepsilon\right\} \text { for } j \in\{1, \ldots, p\}, \\
\Theta_{3} & \equiv\left\{(\gamma(x)-G(x))^{\prime} \beta_{2}:\|\gamma-G\|_{\alpha, p} \leq \varepsilon\right\}, \\
\Theta_{4} & \equiv\left\{h(\tau, d, z, x ; \widetilde{b})-w^{\prime} \beta:\|\gamma-G\|_{\alpha, p} \leq \varepsilon,\|\widetilde{b}-\widetilde{\beta}\|_{E} \leq \delta\right\} .
\end{aligned}
$$

Let $n_{i}(\epsilon) \equiv \log N_{[]}\left(\epsilon, \Theta_{i}, L_{1}(P)\right)$ for $i \in\{1,2,3,4\}$ and $n_{k, j}(\epsilon) \equiv \log N_{[]}\left(\epsilon, \Theta_{k, j}, L_{1}(P)\right)$ for $(k, j) \in\{2,3\} \times\{1, \ldots, p\}$. Let $\psi \equiv \sqrt{\max \{\delta, \varepsilon\}}$.

Since $\Theta_{1}$ is a pointwise Lipschitz class of functions with envelope $\|\widetilde{w}\|_{E} \delta$. By condition $\mathrm{C} 8, E\left(\|\widetilde{w}\|_{E}\right)$ is finite. Thus applying Theorem 2.7.11 of van der Vaart and Wellner (1996, p. 164), we have that

$$
n_{1}\left(\epsilon^{2}\right) \lesssim \frac{q}{\alpha} \log \left(\delta / \epsilon^{2}\right) \lesssim \delta^{q / \alpha} \epsilon^{-2 q / \alpha} \lesssim \psi^{2 q / \alpha} \epsilon^{-2 q / \alpha}
$$

Note that for any norm $\|\cdot\|$, any fixed real valued $c$, any class of functions $\digamma$, it is straightforward to verify that

$$
\begin{aligned}
& N_{[]}(\epsilon, c \digamma,\|\cdot\|)=1 \text { for } c=0 \\
& N_{[]}(\epsilon, c \digamma,\|\cdot\|) \leq N_{[]}(\epsilon /|c|, \digamma,\|\cdot\|) \text { for } c \neq 0
\end{aligned}
$$

where $c \digamma \equiv\{c f: f \in \digamma\}$.

Using this fact, we have that $n_{2, j}\left(\epsilon^{2}\right)=\log N_{[]}\left(\epsilon^{2} /(\varepsilon \delta), \widetilde{\Lambda}_{j} \mathbf{B}_{j}(\delta, \varepsilon), L_{1}(P)\right)$ and $n_{3, j}\left(\epsilon^{2}\right)=0$ for $\beta_{2, j}=0$ and $n_{3, j}\left(\epsilon^{2}\right) \leq \log N_{[]}\left(\epsilon^{2} /\left(\varepsilon\left|\beta_{2, j}\right|\right), \widetilde{\Lambda}_{j}(\varepsilon), L_{1}(P)\right)$ for $\beta_{2, j} \neq 0$. Hence for sufficiently small $\delta$ and $\varepsilon$ (e.g., $\delta<1$ and $\varepsilon<1$ ) and by (C.8), it follows that

$$
n_{2, j}\left(\epsilon^{2}\right) \leq \log N_{[]}\left(\epsilon^{2} \psi^{-2}, \widetilde{\Lambda}_{j} \mathbf{B}_{j}(\delta, \varepsilon), L_{1}(P)\right) \lesssim \psi^{2 q / \alpha} \epsilon^{-2 q / \alpha} .
$$

Using similar arguments, we can also deduce that $n_{3, j}\left(\epsilon^{2}\right) \lesssim \psi^{2 q / \alpha} \epsilon^{-2 q / \alpha}$. 
By preservation of bracketing metric entropy (see, e.g., Lemma 9.25 of Kosorok (2008, p. 169)), we have that for $i \in\{2,3\}$,

$$
n_{i}(\epsilon) \leq n_{i, p}\left(\epsilon 2^{1-p}\right)+\sum_{j=1}^{p-1} n_{i, j}\left(\epsilon 2^{-j}\right)
$$

and $n_{4}(\epsilon) \leq n_{1}(\epsilon / 2)+n_{2}(\epsilon / 4)+n_{3}(\epsilon / 4)$. Therefore by the bounds derived above, it follows that $n_{2}\left(\epsilon^{2}\right) \lesssim \psi^{2 q / \alpha} \epsilon^{-2 q / \alpha}, n_{3}\left(\epsilon^{2}\right) \lesssim \psi^{2 q / \alpha} \epsilon^{-2 q / \alpha}$ and also $n_{4}\left(\epsilon^{2}\right) \lesssim \psi^{2 q / \alpha} \epsilon^{-2 q / \alpha}$.

Now let $f_{1}^{L} \leq f_{1}^{U}, \ldots, f_{N_{[]}\left(\epsilon^{2}, \Theta_{3}, L_{1}(P)\right)}^{L} \leq f_{N_{[]}\left(\epsilon^{2}, \Theta_{3}, L_{1}(P)\right)}^{U}$ and $g_{1}^{L} \leq g_{1}^{U}, \ldots, g_{N_{[]}\left(\epsilon^{2}, \Theta_{4}, L_{1}(P)\right)}^{L} \leq$ $g_{N_{[]}\left(\epsilon^{2}, \Theta_{4}, L_{1}(P)\right)}^{U}$ be the $\epsilon^{2}$-brackets with bracket length defined by $L_{1}(P)$ for the spaces $\Theta_{3}$ and $\Theta_{4}$, respectively. For $1 \leq k \leq N_{[]}\left(\epsilon^{2}, \Theta_{3}, L_{1}(P)\right)$ and $1 \leq j \leq N_{[]}\left(\epsilon^{2}, \Theta_{4}, L_{1}(P)\right)$, define

$$
\begin{aligned}
m_{j k}^{L}(\tau, d, z, x) \equiv & \tau d\left[1\left\{w^{\prime} \beta+g_{j}^{L}(z, x)>0\right\}-1\left\{w^{\prime} \beta+f_{k}^{U}(z, x)>0\right\}\right] \\
& +\tau(1-d)\left[1\left\{w^{\prime} \beta+g_{j}^{U}(z, x) \leq 0\right\}-1\left\{w^{\prime} \beta+f_{k}^{L}(z, x) \leq 0\right\}\right], \\
m_{j k}^{U}(\tau, d, z, x) \equiv & \tau d\left[1\left\{w^{\prime} \beta+g_{j}^{U}(z, x)>0\right\}-1\left\{w^{\prime} \beta+f_{k}^{L}(z, x)>0\right\}\right] \\
& +\tau(1-d)\left[1\left\{w^{\prime} \beta+g_{j}^{L}(z, x) \leq 0\right\}-1\left\{w^{\prime} \beta+f_{k}^{U}(z, x) \leq 0\right\}\right] .
\end{aligned}
$$

Note that

$$
0 \leq m_{j k}^{U}-m_{j k}^{L} \leq 2\left[1\left\{g_{j}^{L} \leq-w^{\prime} \beta<g_{j}^{U}\right\}+1\left\{f_{k}^{L} \leq-w^{\prime} \beta<f_{k}^{U}\right\}\right]
$$

Thus

$$
E\left(m_{j k}^{U}-m_{j k}^{L}\right)^{2} \leq 12 P\left(g_{j}^{L} \leq-w^{\prime} \beta<g_{j}^{U}\right)+4 P\left(f_{k}^{L} \leq-w^{\prime} \beta<f_{k}^{U}\right)
$$

By condition $\mathrm{C} 1$ and given $(\widetilde{z}, x)$, the mapping $z_{1} \longmapsto w^{\prime} \beta$ is one-to-one. Hence by condition $\mathrm{C} 7$, the density of $w^{\prime} \beta$ conditional on $(\widetilde{z}, x)$ is bounded and by (C.10), it then follows that $\left\|m_{j k}^{U}-m_{j k}^{L}\right\|_{L_{2}(P)} \lesssim \epsilon$. Moreover for each $\widetilde{m}_{\widetilde{b}_{, \gamma}} \in \digamma_{\delta, \varepsilon_{N}}$, there is a bracket $\left[m_{j k}^{L}, m_{j k}^{U}\right]$ in which it lies. Therefore,

$$
\log N_{[]}\left(\epsilon, \digamma_{\delta, \varepsilon_{N}}, L_{2}(P)\right) \lesssim n_{3}\left(\epsilon^{2}\right)+n_{4}\left(\epsilon^{2}\right) \lesssim \psi^{2 q / \alpha} \epsilon^{-2 q / \alpha}
$$


Replacing $(\theta, h)$ and $\theta^{*}$ with $\left(\left(\beta_{1,1}, \widetilde{b}\right), \gamma\right)$ and $\left(\beta_{1,1}, \widetilde{\beta}\right)$, respectively in the definition of $\widetilde{S}_{N}$ given by (B.2), we now verify assumption (B.6) in the next lemma.

Lemma 7. For sufficiently small $\delta$ and $\varepsilon$, under conditions $C 1, C 4, C 6, C 7, C 8$ and C10,

$$
E\left[\sup _{\|\widetilde{b}-\widetilde{\beta}\|_{E} \leq \delta,\|\gamma-G\|_{\alpha, p} \leq \varepsilon}\left|\widetilde{S}_{N}(\widetilde{b}, \gamma)\right|\right] \lesssim \frac{\sqrt{\max \{\delta, \varepsilon\}}}{\sqrt{N}} .
$$

Proof. Let $\psi \equiv \sqrt{\max \{\delta, \varepsilon\}}$. By Lemmas 5 and 6 , we have that

$$
\int_{0}^{\left\|M_{\delta, \varepsilon}\right\|_{L_{2}(P)}} \sqrt{\log N_{[]}\left(\epsilon, \digamma_{\delta, \varepsilon}, L_{2}(P)\right)} d \epsilon \lesssim \psi^{q / \alpha} \int_{0}^{a_{2} \psi} \epsilon^{-q / \alpha} d \epsilon \lesssim \psi
$$

where the last inequality follows since $\alpha>q$. Lemma 7 hence follows by applying Corollary 19.35 of van der Vaart (1998, p. 288).

We now prove Theorem 2 .

Proof of Theorem 2. We take $\delta_{N}=N^{-1 / 3}, d_{\Theta}(b, \beta)=\sqrt{c_{1}}\|b-\beta\|_{E}$ and $d_{H}(\gamma, G)=$ $\sqrt{c_{2}}\|\gamma-G\|_{\alpha, p}$ in the application of Lemma 2, where $c_{1}$ and $c_{2}$ are real constants stated in Lemma 4 .

Since $c_{1}>0$, the norm by the metric $d_{\Theta}(\cdot, \cdot)$ is equivalent to the Euclidean norm and thus by Theorem 1, $d_{\Theta}(\widehat{\beta}, \beta)=o_{p}(1)$. Moreover since $c_{2} \geq 0$, assumption C11 implies that $d_{H}(\widehat{G}, G)=O_{p}\left(\varepsilon_{N}\right)$. Given assumption $\mathrm{C} 1$, for sufficiently small $\delta$, we have that $b_{1,1}=\beta_{1,1}$ when $d_{\Theta}(b, \beta) \leq \delta$. Hence for sufficiently small $\delta$ and $\varepsilon_{N}$, by Lemma 4 and noting that $\|\cdot\|_{\alpha, p}$ is stronger than $\|\cdot\|_{\infty}$, assumptions (B.3), (B.4) and (B.5) hold.

By Lemma 7 and by taking $C$ sufficiently large in the definition of $H_{N}(C)$ of Lemma 2, assumption (B.6) also holds with $\phi_{N}(\delta)=\sqrt{\max \left\{\delta, \varepsilon_{N}\right\}}$. Clearly, $\phi_{N}(\delta) \delta^{-\alpha}$ is decreasing for some $\alpha<2$. By assumption C11, $\varepsilon_{N} \leq \delta_{N}$ and thus $\phi_{N}\left(\delta_{N}\right) \leq \sqrt{N} \delta_{N}^{2}$ for every $N$. Therefore, all conditions stated in Lemma 2 are fulfilled and the result of Theorem 2 hence follows. 


\section{Proof of Asymptotic Distribution of $N^{1 / 3}(\widehat{\beta}-\beta)$}

For $C>0$, define the sets

$$
\begin{aligned}
\Theta_{N}(C) & \equiv\left\{b \in \Theta: N^{1 / 3}\|b-\beta\|_{E} \leq C\right\}, \\
H_{N}(C) & \equiv\left\{\gamma \in \Lambda:\|\gamma-G\|_{\alpha, p} \leq C \varepsilon_{N}\right\}
\end{aligned}
$$

where $\varepsilon_{N}$ is the sequence stated in the assumptions of Theorem [3. For each $(b, \gamma)$, define the following recentered empirical and population criterion functions

$$
\begin{aligned}
\bar{S}_{N}(b, \gamma) & \equiv S_{N}(b, \gamma)-S_{N}(\beta, \gamma) \\
\bar{S}(b, \gamma) & \equiv S(b, \gamma)-S(\beta, \gamma) .
\end{aligned}
$$

Clearly, $\widehat{\beta}$ and $\widehat{\beta}_{G}$, defined by (2.6) and (3.2), are still maximizers of the objective functions $\bar{S}_{N}(b, \widehat{G})$ and $\bar{S}_{N}(b, G)$, respectively. Decompose $\bar{S}_{N}(b, \widehat{G})-\bar{S}_{N}(b, G)$ as follows.

$$
\bar{S}_{N}(b, \widehat{G})-\bar{S}_{N}(b, G)=\left[\widetilde{S}_{N}(b, \widehat{G})-\widetilde{S}_{N}(b, G)\right]+[\bar{S}(b, \widehat{G})-\bar{S}(b, G)]
$$

where

$$
\widetilde{S}_{N}(b, \gamma) \equiv \bar{S}_{N}(b, \gamma)-\bar{S}(b, \gamma) .
$$

We shall need the following results.

For $\delta>0$ and $\varepsilon>0$, Consider the local neighborhoods $\Theta(\delta)$ and $H(\varepsilon)$ defined as

$$
\begin{aligned}
\Theta(\delta) & \equiv\left\{b \in \Theta:\|b-\beta\|_{E} \leq \delta\right\}, \\
H(\varepsilon) & \equiv\left\{\gamma \in \Lambda:\|\gamma-G\|_{\alpha, p} \leq \varepsilon\right\} .
\end{aligned}
$$

Recall that $w \equiv(z, G(x)), z \equiv\left(z_{1}, \widetilde{z}\right), \widetilde{w} \equiv(\widetilde{z}, G(x)), \widetilde{b} \equiv\left(\widetilde{b}_{1}, b_{2}\right)$ and $\widetilde{\beta} \equiv\left(\widetilde{\beta}_{1}, \beta_{2}\right)$. Note that for $\delta$ sufficiently small, assumption $\mathrm{C} 1$ implies that $b_{1,1}=\beta_{1,1}$ when $b \in \Theta(\delta)$. Therefore we may assume that $\Theta(\delta)=\left\{b \in \Theta: b_{1,1}=\beta_{1,1}\right.$ and $\left.\widetilde{b} \in \widetilde{\Theta}(\delta)\right\}$ where $\widetilde{\Theta}(\delta) \equiv\left\{\widetilde{b} \in \Upsilon:\|\widetilde{b}-\widetilde{\beta}\|_{E} \leq \delta\right\}$. For any $s=(\tau, d, z, x)$, consider the following 
function

$$
\bar{m}_{\widetilde{b}, \gamma}(z, x) \equiv 1\left\{z_{1} \beta_{1,1}+\widetilde{z}^{\widetilde{b}} \widetilde{b}_{1}+\gamma(x)^{\prime} b_{2}>0\right\}-1\left\{z_{1} \beta_{1,1}+\widetilde{z}^{\prime} \widetilde{\beta}_{1}+\gamma(x)^{\prime} \beta_{2}>0\right\}
$$

Let

$$
\widetilde{m}_{\widetilde{b}, \gamma}(s) \equiv \tau(2 d-1)\left[\bar{m}_{\widetilde{b}, \gamma}(z, x)-\bar{m}_{\widetilde{b}, G}(z, x)\right] .
$$

Define the class of functions

$$
\digamma_{\delta, \varepsilon} \equiv\left\{\widetilde{m}_{\widetilde{b}, \gamma}:(\widetilde{b}, \gamma) \in \widetilde{\Theta}(\delta) \times H(\varepsilon)\right\}
$$

Let $M_{\delta, \varepsilon}$ denote an envelope for $\digamma_{\delta, \varepsilon}$ such that $\left|\widetilde{m}_{\widetilde{b}, \gamma}(s)\right| \leq\left|M_{\delta, \varepsilon}(s)\right|$ for all $s$ and for all $\widetilde{m}_{\widetilde{b}, \gamma} \in \digamma_{\delta, \varepsilon}$.

Lemma 8. Let $\delta$ and $\varepsilon$ be sufficiently small. Given conditions C1, C4, C6 and C10, for some positive real constants $c_{1}$ and $c_{2}$, we can take

$$
M_{\delta, \varepsilon}=2 \times 1\left\{c_{1} \min \{\delta, \varepsilon\} \geq\left|w^{\prime} \beta\right|\right\}
$$

and furthermore,

$$
\left\|M_{\delta, \varepsilon}\right\|_{L_{2}(P)} \leq c_{2} \sqrt{\min \{\delta, \varepsilon\}}
$$

Proof. Note that

$\left|\widetilde{m}_{\widetilde{b}, \gamma}(s)\right| \leq 2 \times 1\left\{\left[\bar{m}_{\widetilde{b}, \gamma}(s)=1\right.\right.$ and $\left.\bar{m}_{\widetilde{b}, G}(s)=-1\right]$ or $\left[\bar{m}_{\widetilde{b}, \gamma}(s)=-1\right.$ and $\left.\left.\bar{m}_{\widetilde{b}, G}(s)=1\right]\right\}$.

Given C1, C6 and C10, there is positive real constant $B$ such that $\max \left\{\|\widetilde{w}\|_{E},\left\|b_{2}\right\|_{E}\right\}<$ $B$ with probability 1 . Hence if $(\widetilde{b}, \gamma) \in \widetilde{\Theta}(\delta) \times H(\varepsilon)$, we have that

$$
\begin{array}{ll} 
& \bar{m}_{\widetilde{b}, \gamma}(s)=1 \\
\Longleftrightarrow \quad & z_{1} \beta_{1,1}+\widetilde{z}^{\top} \widetilde{b}_{1}+\gamma(x)^{\prime} b_{2}>0 \geq z_{1} \beta_{1,1}+\widetilde{z}^{\prime} \widetilde{\beta}_{1}+\gamma(x)^{\prime} \beta_{2} \\
\Longleftrightarrow & \widetilde{w}^{\prime}(\widetilde{b}-\widetilde{\beta})+(\gamma(x)-G(x))^{\prime} b_{2}>-w^{\prime} \beta \geq(\gamma(x)-G(x))^{\prime} \beta_{2} \\
\Longrightarrow & -B(\delta+\varepsilon) \leq w^{\prime} \beta \leq B \varepsilon .
\end{array}
$$


On the other hand,

$$
\begin{array}{ll} 
& \bar{m}_{\widetilde{b}, G}(s)=-1 \\
\Longleftrightarrow \quad & z_{1} \beta_{1,1}+\widetilde{w}^{\prime} \widetilde{b} \leq 0<w^{\prime} \beta \\
\Longleftrightarrow \quad & \widetilde{w}^{\prime}(\widetilde{b}-\widetilde{\beta}) \leq-w^{\prime} \beta<0 \\
\Longrightarrow & 0 \leq w^{\prime} \beta \leq B \delta .
\end{array}
$$

Therefore, the condition $\bar{m}_{\widetilde{b}, \gamma}(s)=1$ and $\bar{m}_{\widetilde{b}, G}(s)=-1$ implies $\left|w^{\prime} \beta\right| \leq B \min \{\delta, \varepsilon\}$. Based on similar arguments, we can verify that the condition $\bar{m}_{\widetilde{b}, \gamma}(s)=-1$ and $\bar{m}_{\widetilde{b}, G}(s)=1$ also implies $\left|w^{\prime} \beta\right| \leq B \min \{\delta, \varepsilon\}$. Therefore, Lemma 8 follows by taking $M_{\delta, \varepsilon}=2 \times 1\left\{\left|w^{\prime} \beta\right| \leq B \min \{\delta, \varepsilon\}\right\}$ and noting that given C4, inequality (D.6) holds for $c_{2}=2 \sqrt{2 c_{1} L}$.

Lemma 9. Given conditions C1, C4, C6, C7, C8 and C10, we have that for sufficiently small $\delta$ and $\varepsilon$,

$$
E\left[\sup _{(b, \gamma) \in \widetilde{\Theta}(\delta) \times H(\varepsilon)}\left|\widetilde{S}_{N}(b, \gamma)-\widetilde{S}_{N}(b, G)\right|\right] \lesssim N^{-1 / 2}(\max \{\delta, \varepsilon\})^{\frac{q}{2 \alpha}}(\min \{\delta, \varepsilon\})^{\frac{\alpha-q}{2 \alpha}}
$$

Proof. Define the following two classes of functions

$$
\begin{aligned}
A_{\delta, \varepsilon} & \equiv\left\{\tau(2 d-1) \bar{m}_{\widetilde{b}, \gamma}(z, x):(\widetilde{b}, \gamma) \in \widetilde{\Theta}(\delta) \times H(\varepsilon)\right\} \\
B_{\delta, \varepsilon} & \equiv\left\{\tau(2 d-1) \bar{m}_{\widetilde{b}, G}(z, x): \widetilde{b} \in \widetilde{\Theta}(\delta)\right\} .
\end{aligned}
$$

Using Lemma 9.25 of Kosorok (2008, p. 169)), we have that

$$
\log N_{[]}\left(\epsilon, \digamma_{\delta, \varepsilon}, L_{2}(P)\right) \leq \log N_{[]}\left(\epsilon / 2, A_{\delta, \varepsilon}, L_{2}(P)\right)+\log N_{[]}\left(\epsilon / 2, B_{\delta, \varepsilon}, L_{2}(P)\right)
$$

Let $\psi \equiv \sqrt{\max \{\delta, \varepsilon\}}$. By Lemma 6 , we have that for sufficiently small $\delta$ and $\varepsilon$,

$$
\log N_{[]}\left(\epsilon, A_{\delta, \varepsilon}, L_{2}(P)\right) \lesssim \psi^{2 q / \alpha} \epsilon^{-2 q / \alpha}
$$


Furthermore by simplifying proof of Lemma 6, it is straightforward to verify that

$$
\log N_{[]}\left(\epsilon, B_{\delta, \varepsilon}, L_{2}(P)\right) \lesssim \psi^{2 q / \alpha} \epsilon^{-2 q / \alpha}
$$

and thus

$$
\log N_{\square}\left(\epsilon, \digamma_{\delta, \varepsilon}, L_{2}(P)\right) \lesssim \psi^{2 q / \alpha} \epsilon^{-2 q / \alpha} .
$$

Using inequality (D.7) and Lemmas 8, we have that

$\int_{0}^{\left\|M_{\delta, \varepsilon}\right\|_{L_{2}(P)}} \sqrt{\log N_{[]}\left(\epsilon, \digamma_{\delta, \varepsilon}, L_{2}(P)\right)} d \epsilon \lesssim \psi^{\frac{q}{\alpha}} \int_{0}^{c_{2} \sqrt{\min \{\delta, \varepsilon\}}} \epsilon^{-\frac{q}{\alpha}} d \epsilon \lesssim \psi^{\frac{q}{\alpha}}(\min \{\delta, \varepsilon\})^{\frac{\alpha-q}{2 \alpha}}$

where the last inequality follows from the assumption $\alpha>q$. Lemma 9 hence follows by applying Corollary 19.35 of van der Vaart (1998, p. 288).

We now prove Theorem 3 ,

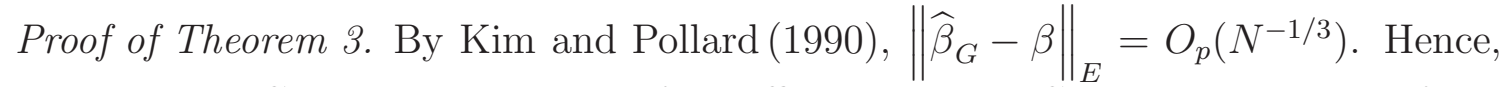
by condition $\mathrm{C} 11$ and Theorem 2 , for sufficiently large $C>0$, probability of the event that $\widehat{\beta} \in \Theta_{N}(C), \widehat{\beta}_{G} \in \Theta_{N}(C)$ and $\widehat{G} \in H_{N}(C)$ can be made arbitrarily close to 1 . Thus to show the theorem, it suffices to establish that for any fixed sufficiently large $C>0$,

$$
\sup _{b \in \Theta_{N}(C)}\left|\bar{S}_{N}(b, \widehat{G})-\bar{S}_{N}(b, G)\right|=o_{p}\left(N^{-2 / 3}\right) .
$$

Given (D.8), we have that

$$
\begin{aligned}
\bar{S}_{N}(\widehat{\beta}, G) & \geq \bar{S}_{N}(\widehat{\beta}, \widehat{G})-o_{p}\left(N^{-2 / 3}\right) \\
& \geq \bar{S}_{N}\left(\widehat{\beta}_{G}, \widehat{G}\right)-o_{p}\left(N^{-2 / 3}\right) \\
& \geq \bar{S}_{N}\left(\widehat{\beta}_{G}, G\right)-o_{p}\left(N^{-2 / 3}\right)
\end{aligned}
$$

where the first and third inequalities follow from (D.8) and the second inequality follows from the definition of $\widehat{\beta}$. Therefore by Theorem 1.1 of Kim and Pollard (1990), $N^{1 / 3}(\widehat{\beta}-\beta)$ and $N^{1 / 3}\left(\widehat{\beta}_{G}-\beta\right)$ are asymptotically equivalent in distribution. 
We now verify equation (D.8). Given the decomposition (D.1), it suffices to show that

$$
\begin{aligned}
& E\left[\sup _{(b, \gamma) \in \Theta_{N}(C) \times H_{N}(C)}\left|\widetilde{S}_{N}(b, \gamma)-\widetilde{S}_{N}(b, G)\right|\right]=o\left(N^{-2 / 3}\right), \\
& \sup _{b \in \Theta_{N}(C)}|\bar{S}(b, \widehat{G})-\bar{S}(b, G)|=o_{p}\left(N^{-2 / 3}\right) .
\end{aligned}
$$

Equation (D.9) concerns stochastic equicontinuity of the local recentered process $N^{2 / 3} \widetilde{S}_{N}(b, \gamma)$ indexed by $(b, \gamma) \in \Theta_{N}(C) \times H_{N}(C)$. It is satisfied by setting $\delta=$ $C N^{-1 / 3}$ and $\varepsilon=C \varepsilon_{N}$ in the definition of sets $\Theta(\delta)$ and $H(\varepsilon)$ given by (D.2) and (D.3) and by invoking Lemma 9 with the assumptions $\varepsilon_{N}=o\left(N^{-1 / 3}\right)$ and $\alpha>q$.

We now verify equation (D.10). Note that for $N$ sufficiently large, if $b \in \Theta_{N}(C)$, then $b_{1,1}=\beta_{1,1}$ under condition $\mathrm{C} 1$. Let

$$
\bar{S}_{1}(\widetilde{b}, \gamma) \equiv S_{1}(\widetilde{b}, \gamma)-S_{1}(\widetilde{\beta}, \gamma)
$$

where $S_{1}(\widetilde{b}, \gamma)$ is defined by (C.1). Hence it suffices to verify

$$
\sup _{\widetilde{b} \in \widetilde{\Theta}_{N}(C)}\left|\bar{S}_{1}(\widetilde{b}, \widehat{G})-\bar{S}_{1}(\widetilde{b}, G)\right|=o_{p}\left(N^{-2 / 3}\right)
$$

where $\widetilde{\Theta}_{N}(C) \equiv\left\{\widetilde{b} \in \Upsilon:\|\widetilde{b}-\widetilde{\beta}\|_{E} \leq C N^{-1 / 3}\right\}$.

Note that the term $\left|\bar{S}_{1}(\widetilde{b}, \widehat{G})-\bar{S}_{1}(\widetilde{b}, G)\right|$ is bounded above by

$$
\left|S_{1}(\widetilde{\beta}, \widehat{G})-S_{1}(\widetilde{\beta}, G)\right|+\left|\left[S_{1}(\widetilde{b}, \widehat{G})-S_{1}(\widetilde{\beta}, G)\right]-\left[S_{1}(\widetilde{b}, G)-S_{1}(\widetilde{\beta}, G)\right]\right| .
$$

Since $\varepsilon_{N}=o\left(N^{-1 / 3}\right)$, by C11 we have that $\|\widehat{G}-G\|_{\infty}=o_{p}\left(N^{-1 / 3}\right)$ because the norm $\|\cdot\|_{\alpha, p}$ is stronger than the sup norm $\|\cdot\|_{\infty}$. Hence by Lemma 4 , the first term of the sum (D.11) is $o_{p}\left(N^{-2 / 3}\right)$ and

$$
S_{1}(\widetilde{b}, \widehat{G})-S_{1}(\widetilde{\beta}, G)=-\left(A_{1}(\widetilde{b})+A_{2}(\widehat{G})+e(\widetilde{b}, \widehat{G})\right)+o_{p}\left(N^{-2 / 3}\right)
$$


where the terms $A_{1}(\widetilde{b}), A_{2}(\widehat{G})$ and $e(\widetilde{b}, \widehat{G})$ are given by (C.2), (C.3) and (C.4), respectively. Using the proof of Lemma 4, it is also straightforward to verify that

$$
S_{1}(\widetilde{b}, G)-S_{1}(\widetilde{\beta}, G)=-A_{1}(\widetilde{b})+o_{p}\left(N^{-2 / 3}\right) .
$$

Since $\|\widehat{G}-G\|_{\infty}=o_{p}\left(N^{-1 / 3}\right)$ and $\widetilde{b} \in \widetilde{\Theta}_{N}(C)$, we have that $A_{2}=o_{p}\left(N^{-2 / 3}\right)$ and $e(\widetilde{b}, \widehat{G})=o_{p}\left(N^{-2 / 3}\right)$. Putting together (D.12) and (D.13), it follows that the second term of the sum (D.11) is also $o_{p}\left(N^{-2 / 3}\right)$ and therefore equation (D.10) holds.

\section{References}

[1] Abrevaya, J., and Huang, J. (2005), "On the bootstrap of the maximum score estimator", Econometrica, 73 (4), 1175-1204.

[2] Ackerberg, D., X. Chen, and J. Hahn (2012), "A Practical Asymptotic Variance Estimator for Two-Step Semiparametric Estimators", Review of Economics and Statistics 94, 481-498.

[3] Ahn, H., (1995), "nonparametric Two-Stage Estimation of Conditional Choice Probabilities in a Binary Choice Model under Uncertainty", Journal of Econometrics, 67, 337-378.

[4] Ahn, H., (1997), "Semiparametric Estimation of a Single-Index Model with Nonparametrically Generated Regressors", Econometric Theory, 13, 3-31.

[5] Ahn, H. and C. F. Manski (1993), "Distribution Theory for the Analysis of Binary Choice under Uncertainty with Nonparametric Estimation of Expectations", Journal of Econometrics, 56, 291-321.

[6] Andrews, D. W. K. (1995), "Nonparametric Kernel Estimation for Semiparametric Models", Econometric Theory, 11, 560-596.

[7] Aradillas-Lopez, A. (2012), "Pairwise Difference Estimation of Incomplete Information Games", Journal of Econometrics, 168, 120-140. 
[8] Arellano, M. and Honoré, B. (2001), "Panel data models: some recent developments", In: J.J. Heckman and E. Leamer. (eds), Handbook of Econometrics, Vol. 5, 3229-3296.

[9] Bierens, H. J. (1983), "Uniform Consistency of Kernel Estimators of a Regression Function Under Generalized Conditions", Journal of the American Statistical Association, 78, 699-707.

[10] Bierens, H. J. (1987), "Kernel Estimators of Regression Functions", in Truman F.Bewley (ed.), Advances in Econometrics: Fifth World Congress, Vol. 1, Cambridge University Press, 99-144.

[11] Brown, Bryan W., Walker, Mary Beth (1989), "The random utility hypothesis and inference in demand systems", Econometrica, 57, 815-829.

[12] Chen, X., Linton, O. and van Keilegom, I. (2003), "Estimation of semiparametric models when the criterion function is not smooth", Econometrica, 71, 1591-1608.

[13] Chen, X. and Pouzo, D. (2009), "Efficient Estimation of Semiparametric Conditional Moment Models with Possibly Nonsmooth Residuals", Journal of Econometrics, 152, 46-60.

[14] Chen, X. and Pouzo, D. (2012), "Estimation of Nonparametric Conditional Moment Models With Possibly Nonsmooth Generalized Residuals", Econometrica, 80, 277-321.

[15] Chen, X., Hahn, J., Liao, Z. and Ridder G. (2013), "Nonparametric Two-Step Sieve M Estimation and Inference", Working paper.

[16] Coppejans, M. (2001), "Estimation of the Binary Response Model Using a Mixture of Distributions Estimator (MOD)", Journal of Econometrics, 102, 231-261.

[17] Cosslett, S. R. (1983), "Distribution-free Maximum Likelihood Estimator of the Binary Choice Model", Econometrica, 51, 765-782. 
[18] Delgado, M.A., Rodriguez-Poo, J.M.,and Wolf, M. (2001), "Subsampling inference in cube root asymptotics with an application to Manski's maximum score estimator", Economics Letters, 73 (2), 241-250.

[19] Efromovich, S. (2001), "Density Estimation Under Random Censorship and Order Restrictions: From Asymptotic to Small Samples," Journal of the American Statistical Association, 96, 667-684.

[20] Escanciano, J., D. Jacho-Chávez, and A. Lewbel (2012), "Identification and Estimation of Semiparametric Two Step Models", Working Paper.

[21] Escanciano, J., D. Jacho-Chávez, and A. Lewbel (2013), "Uniform Convergence of Weighted Sums of Non- and Semi-parametric Residuals for Estimation and Testing", Working Paper.

[22] Florios, K. and Skouras, S. (2008), "Exact computation of max weighted score estimators", Journal of Econometrics, 146, 86-91.

[23] Fox, J. T. (2007), "Semiparametric estimation of multinomial discrete-choice models using a subset of choices", RAND Journal of Economics, 38, 1002-1019.

[24] Hahn, J., and G. Ridder (2013), "The Asymptotic Variance of Semi-parametric Estimators with Generated Regressors", Econometrica, 81, 315-340.

[25] Horowitz, J. (1992), "A Maximum Score Estimator for the Binary Response Model", Econometrica, 60, 505-531.

[26] Ichimura, H. (1993), "Semiparametric Least Squares (SLS) and Weighted SLS Estimation of Single Index Models", Journal of Econometrics, 58, 71-120.

[27] Ichimura, H. and Lee, S. (2010), "Characterization of the asymptotic distribution of semiparametric M-estimators", Journal of Econometrics, 159, 252-266.

[28] Jun, S. J., Pinkse, J. and Wan, Y. (2013), "Classical Laplace Estimation for $\sqrt[3]{n}$-Consistent Estimators: Improved Convergence Rates and Rate-Adaptive 
Inference", Working paper, Department of Economics, Pennsylvania State University.

[29] Kim, J. and Pollard, D. (1990), "Cube root asymptotics", Annals of Statistics, 18, 191-219.

[30] Klein, R. and Spady, R. (1993), "An Efficient Semiparametric Estimator for Binary Response Models", Econometrica, 61, 387-421.

[31] Kosorok, M. R. (2008), Introduction to Empirical Processes and Semiparametric Inference, Springer, New York.

[32] Lee, S. M. S., and Pun, M.C. (2006), "On $m$ out of $n$ bootstrapping for nonstandard M-estimation", Journal of the American Statistical Association, 101, 1185-1197.

[33] Lewbel, A. (2000), "Semiparametric qualitative response model estimation with unknown heteroscedasticity or instrumental variables", Journal of Econometrics, 97, 145-177.

[34] Mammen, E., C. Rothe, and M. Schienle (2013), "Semiparametric Estimation with Generated Covariates", Working Paper.

[35] Manski, C. F. (1975), "Maximum score estimation of the stochastic utility model of choice," Journal of Econometrics, 3, 205-228.

[36] Manski, C. F. (1985), "Semiparametric analysis of discrete response. Asymptotic properties of the maximum score estimator", Journal of Econometrics, 27, 313333.

[37] Manski, C. F. (1991), "Nonparametric Estimation of Expectations in the Analysis of Discrete Choice under Uncertainty" in W. Barnett, J. Powell, and G. Tauchen, (editors), Nonparametric and Semiparametric Methods in Econometrics and Statistics, Cambridge: Cambridge Press. 
[38] Manski, C. F. (1993), "Dynamic choice in social settings : Learning from the experiences of others", Journal of Econometrics, 58, 121-136.

[39] Marron, J. S. and M. P. Wand (1992), "Exact Mean Integrated Squared Error", Annals of Statistics, 20, 712-736.

[40] Newey, W.K. and McFadden, D.L. (1994), "Large Sample Estimation and Hypothesis Testing", In: Engle R. F. and McFadden, D. (eds), Handbook of Econometrics, Vol. 4, 2111-2245.

[41] Newey, W.K. (1997), "Convergence rates and asymptotic normality for series estimators", Journal of Econometrics, 79, 147-168.

[42] Powell, J. L., Stock, J. H., and T. M. Stoker (1989), "Semiparametric Estimation of Index Coefficients," Econometrica, 57, 1403-1430.

[43] Van der Vaart, A. W. and Wellner, J. A. (1996), Weak Convergence and Empirical Processes: With Applications to Statistics, Springer, New York.

[44] Van der Vaart, A. W. (1998), Asymptotic Statistics, Cambridge University Press. 
Table 1 : Simulation Results for $\widehat{\beta}_{2, \text { Single }}$ and $\widehat{\beta}_{2, O L S}$ (linear $G$ )

\begin{tabular}{cccccc}
\hline \hline$N$ & Bias & RMSE & Median & mean AD & median AD \\
300 & -0.112 & 0.410 & 0.894 & 0.329 & 0.288 \\
500 & -0.061 & 0.352 & 0.916 & 0.285 & 0.247 \\
1000 & -0.031 & 0.264 & 0.961 & 0.211 & 0.182 \\
300 & -0.122 & 0.478 & 0.856 & 0.381 & 0.326 \\
500 & -0.070 & 0.376 & 0.908 & 0.304 & 0.259 \\
1000 & -0.033 & 0.301 & 0.952 & 0.240 & 0.211
\end{tabular}

Table 2 : Simulation Results for $\widehat{\beta}_{2, \text { Single }}$ and $\widehat{\beta}_{2, O L S}$ (nonlinear $G$ )

\begin{tabular}{cccccc}
\hline \hline$N$ & Bias & RMSE & $\begin{array}{c}\text { Median } \\
\text { Single-stage estimation }\end{array}$ & mean AD \\
300 & -0.056 & 0.330 & 0.918 & 0.262 & 0.216 \\
500 & -0.044 & 0.277 & 0.942 & 0.220 & 0.184 \\
1000 & -0.020 & 0.212 & 0.966 & 0.169 & 0.139 \\
300 & -0.394 & 0.489 & 0.577 & 0.431 & 0.432 \\
500 & -0.413 & 0.469 & 0.568 & 0.424 & 0.432 \\
1000 & -0.400 & 0.435 & 0.587 & 0.402 & 0.412
\end{tabular}


Table 3 : Simulation Results for $\widehat{\beta}_{2, \text { Kernel_sth }}$ (linear $G$ )

\begin{tabular}{|c|c|c|c|c|c|}
\hline \multicolumn{6}{|c|}{$\begin{array}{c}c \quad \text { Bias RMSE Median mean AD median A } \\
\text { Two-stage estimation : kernel first stage }(N=300)\end{array}$} \\
\hline 5.4 & -0.063 & 0.639 & 0.868 & 0.502 & 0.417 \\
\hline & 0.041 & 0.653 & & & 0.400 \\
\hline & 0.138 & 0.718 & 1.067 & 0.544 & 0.427 \\
\hline \multicolumn{6}{|c|}{ Two-stage estimation: kernel first stage $(N=500)$} \\
\hline & -0.046 & 0.501 & 0.908 & 0.388 & 0.314 \\
\hline & 0.056 & 0.518 & 0.992 & & 0.307 \\
\hline 5.8 & 0.171 & 0.584 & 1.096 & 0.435 & 0.331 \\
\hline \multicolumn{6}{|c|}{ Two-stage estimation : kernel first stage $(N=1000)$} \\
\hline & -0.087 & 0.389 & 0.887 & 0.311 & 0.266 \\
\hline & 0.008 & 0.380 & 0.992 & 0.307 & 0.264 \\
\hline .8 & 0.111 & 0.424 & 1.086 & 0.334 & 0.278 \\
\hline
\end{tabular}

Table 4 : Simulation Results for $\widehat{\beta}_{2, \text { Kernel_sth }}$ (nonlinear $G$ )

\begin{tabular}{cccccc}
\hline \hline$c$ & Bias & RMSE & Median & mean AD & median AD \\
5.8 & -0.071 & 0.480 & 0.918 & 0.380 & 0.328 \\
6 & 0.053 & 0.523 & 1.028 & 0.408 & 0.340 \\
6.2 & 0.147 & 0.577 & 1.132 & 0.448 & 0.372 \\
5.8 & Two-stage estimation : kernel first stage & $(N=500)$ \\
5.0 .066 & 0.400 & 0.906 & 0.316 & 0.264 \\
6 & 0.030 & 0.408 & 1.004 & 0.323 & 0.268 \\
6.2 & 0.103 & 0.457 & 1.062 & 0.355 & 0.288 \\
5.8 & -0.125 & 0.298 & 0.865 & 0.243 & 0.211 \\
6 & -0.028 & 0.286 & 0.956 & 0.230 & 0.192 \\
6.2 & 0.059 & 0.320 & 1.038 & 0.251 & 0.211
\end{tabular}


Table 5 : Simulation Results for $\widehat{\beta}_{2, \text { Kernel_2nd }}$ (linear $G$ )

\begin{tabular}{|c|c|c|c|c|c|}
\hline \multirow{2}{*}{\multicolumn{6}{|c|}{$\begin{array}{c}c \quad \text { Bias RMSE Median mean AD median AD } \\
\text { Two-stage estimation : kernel first stage }(N=300)\end{array}$}} \\
\hline & & & & & \\
\hline 0.6 & -0.172 & 0.483 & 0.803 & 0.392 & 0.345 \\
\hline 0.8 & -0.122 & 0.502 & 0.865 & 0.395 & 0.328 \\
\hline 1 & -0.088 & 0.510 & 0.896 & 0.401 & 0.333 \\
\hline \multicolumn{6}{|c|}{ Two-stage estimation : kernel first stage $(N=500)$} \\
\hline 0.6 & -0.111 & 0.391 & 0.880 & 0.315 & 0.276 \\
\hline 0.8 & -0.073 & 0.394 & 0.913 & 0.316 & 0.268 \\
\hline 1 & -0.037 & 0.408 & 0.937 & 0.326 & 0.280 \\
\hline \multicolumn{6}{|c|}{ Two-stage estimation : kernel first stage $(N=1000)$} \\
\hline 0.6 & -0.054 & 0.305 & 0.923 & 0.247 & 0.216 \\
\hline 0.8 & -0.028 & 0.301 & 0.956 & 0.242 & 0.211 \\
\hline 1 & 0.002 & 0.313 & 0.980 & 0.250 & 0.216 \\
\hline
\end{tabular}

Table 6 : Simulation Results for $\widehat{\beta}_{2, \text { Kernel_2nd }}$ (nonlinear $G$ )

\begin{tabular}{cccccc}
\hline \hline$c$ & Bias & RMSE & Median & mean AD & median AD \\
\multicolumn{7}{c}{ Two-stage estimation : kernel first stage } & $(N=300)$ \\
0.6 & -0.112 & 0.440 & 0.865 & 0.347 & 0.297 \\
0.8 & -0.057 & 0.443 & 0.918 & 0.351 & 0.302 \\
1 & -0.009 & 0.469 & 0.968 & 0.370 & 0.316 \\
\multicolumn{5}{c}{ Two-stage estimation : kernel first stage } & $(N=500)$ \\
0.6 & -0.077 & 0.366 & 0.918 & 0.291 & 0.244 \\
0.8 & -0.040 & 0.382 & 0.932 & 0.302 & 0.254 \\
1 & -0.010 & 0.397 & 0.966 & 0.313 & 0.264 \\
0.6 & -0.037 & 0.272 & 0.952 & 0.218 & 0.182 \\
0.8 & -0.012 & 0.272 & 0.980 & 0.218 & 0.192 \\
1 & 0.036 & 0.286 & 1.028 & 0.230 & 0.201
\end{tabular}


Table 7 : Comparison of Empirical Distribution Functions (8th order kernel)
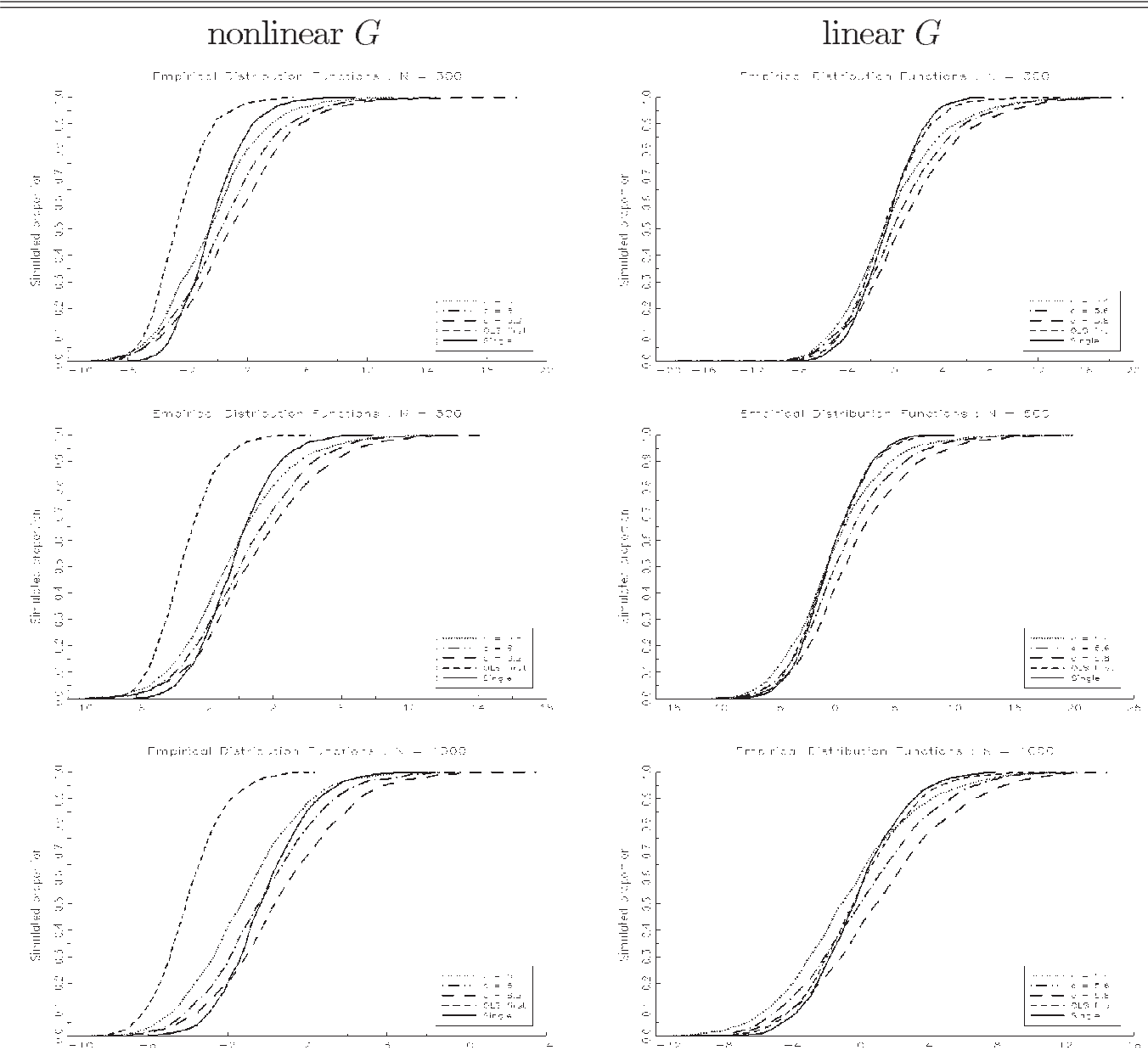
Table 8 : Comparison of Empirical Distribution Functions (2nd order kernel)
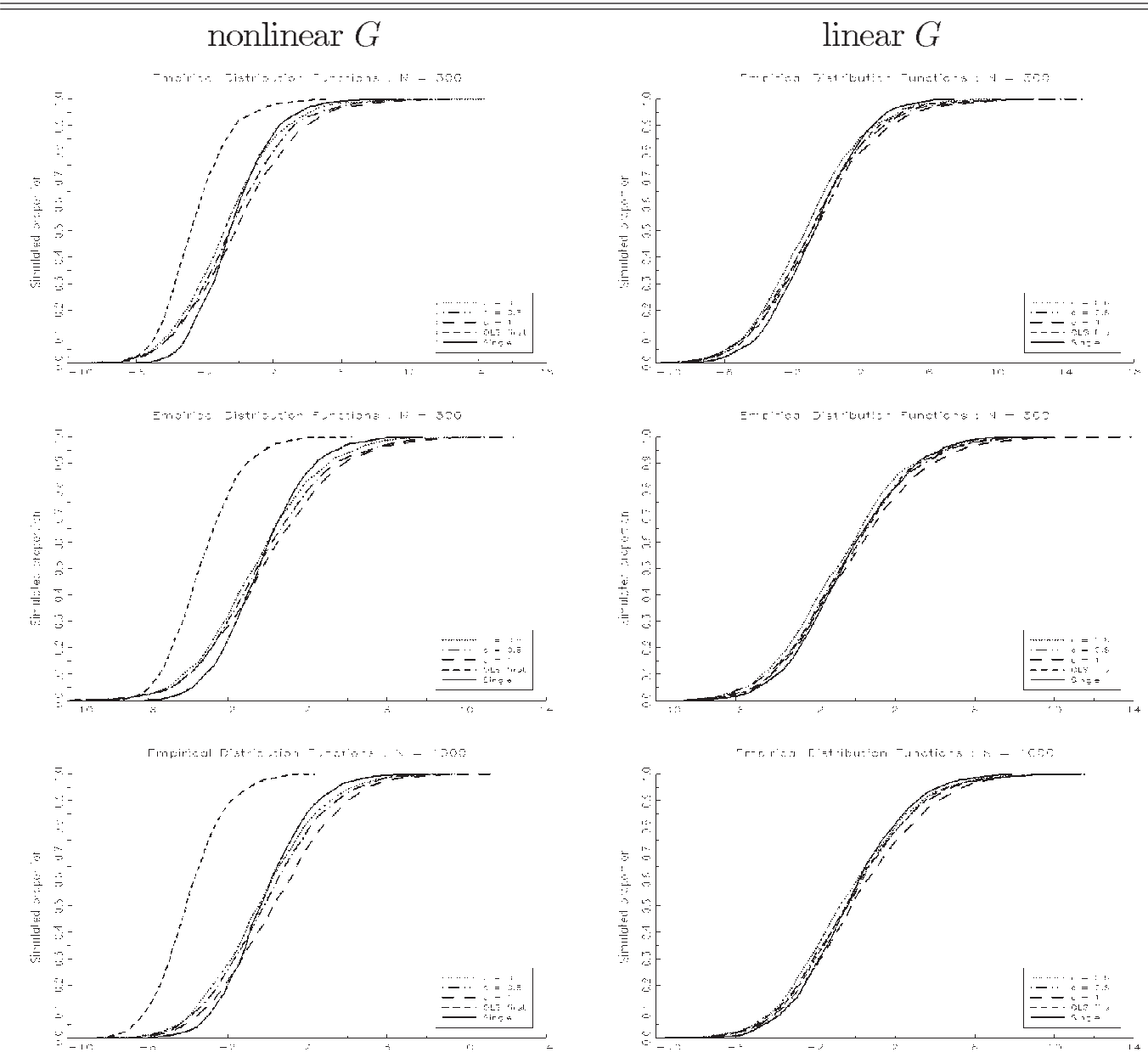